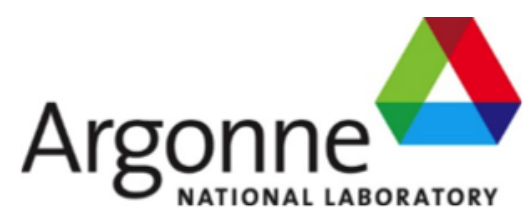

\title{
Initial development and extension of EPP methods to Grade 91
}

Applied Materials Division 
About Argonne National Laboratory

Argonne is a U.S. Department of Energy laboratory managed by UChicago Argonne, LLC under contract DE-AC02-06CH11357. The Laboratory's main facility is outside Chicago, at 9700 South Cass Avenue, Argonne, Illinois 60439. For information about Argonne and its pioneering science and technology programs, see www.anl.gov.

\section{DOCUMENT AVAILABILITY}

Online Access: U.S. Department of Energy (DOE) reports produced after 1991 and a growing number of pre-1991 documents are available free at OSTI.GOV (http://www.osti.gov/), a service of the U.S. Dept. of Energy's Office of Scientific and Technical Information

Reports not in digital format may be purchased by the public from the

National Technical Information Service (NTIS):

U.S. Department of Commerce

National Technical Information Service

5301 Shawnee Rd

Alexandria, VA 22312

www.ntis.gov

Phone: (800) 553-NTIS (6847) or (703) 605-6000

Fax: (703) 605-6900

Email: orders@ntis.gov

Reports not in digital format are available to DOE and DOE contractors from the Office of Scientific and Technical Information (OSTI)

U.S. Department of Energy

Office of Scientific and Technical Information

P.O. Box 62

Oak Ridge, TN 37831-0062

www.osti.gov

Phone: (865) 576-8401

Fax: (865) 576-5728

Email: reports@osti.gov

\section{Disclaimer}

This report was prepared as an account of work sponsored by an agency of the United States Government. Neither the United States Government nor any agency thereof, nor UChicago Argonne, LLC, nor any of their employees or officers, makes any warranty, express or implied, or assumes any legal liability or responsibility for the accuracy, completeness, or usefulness of any information, apparatus, product, or process disclosed, or represents that its use would not infringe privately owned rights. Reference herein to any specific commercial product, process, or service by trade name, trademark, manufacturer, or otherwise, does not necessarily constitute or imply its endorsement, recommendation, or favoring by the United States Government or any agency thereof. The views and opinions of document authors expressed herein do not necessarily state or reflect those of the United States Government or any agency thereof, Argonne National Laboratory, or UChicago Argonne, LLC. 


\section{Initial development and extension of EPP methods to Grade 91}

Applied Materials Division

Argonne National Laboratory

July 2018

Prepared by

M. C. Messner, Argonne National Laboratory

T.-L. Sham, Argonne National Laboratory 



\section{Abstract}

This report describes modifications to the existing elastic perfectly plastic (EPP) analysis ASME Nuclear Code Cases N-861 and N-862 required to apply the Code Case design methods to Grade 91 steel. The critical difference between the existing Code Cases, applicable only to $304 \mathrm{H}$ and $316 \mathrm{H}$ stainless steel, is that they do not account for material cyclic softening. Grade 91 is a softening material at elevated temperature. As such, modifications to the existing Code Case rules are required before they can be conservatively applied to Grade 91. This report describes modified EPP methods applicable to softening materials like Grade 91. Additionally, the report describes the specific technical and administrative changes to the existing EPP Code Cases that will be required to support Grade 91. 

Initial development and extension of EPP methods to Grade 91

July 2018

\section{Table of Contents}

Abstract

Table of Contents $\quad$ iii

List of Figures $\quad$ v

List of Tables vii

1 Introduction 1

2 Verifying design methods using full inelastic analysis 3

2.1 Verification strategy . . . . . . . . . . . . . . . . . . 3

2.2 Reference material model . . . . . . . . . . . . . . . . . . . . . . . . 4

2.2.1 Constitutive model . . . . . . . . . . . . . . . . . . . . . 4

2.2.2 Example response . . . . . . . . . . . . . . . . . . . . 11

2.2 .3 Design data . . . . . . . . . . . . . . . . . . . . . . . . . . . . 11

3 Strain limits 19

3.1 Unmodified Code Case . . . . . . . . . . . . . . . . . . . . . . . 19

3.2 Modified Code Case . . . . . . . . . . . . . . . . . . . . . . . 21

3.3 Isochronous curve softening factors . . . . . . . . . . . . . 27

4 Creep-fatigue 31

5 Proposed modifications to the EPP Code Cases 35

5.1 Strain limits: Code Case N-861 . . . . . . . . . . . . . . . . . . . 35

5.2 Creep-fatigue: Code Case N-862 . . . . . . . . . . . . . . . . 37

6 Conclusions 39

Acknowledgments $\quad 41$

Bibliography

Distribution List $\quad 45$ 



\section{List of Figures}

2.1 Kocks-Mecking master curve for Grade 91 steel, as established from a large experimental database. . . . . . . . . . . . . . . .

2.2 Example simulations of pure fatigue loading comparing the model with the damage model turned on and without damage. The loading conditions are $T=540^{\circ} \mathrm{C}, \Delta \varepsilon=0.01 \mathrm{~mm} / \mathrm{mm}$ full reversed loading, and $\dot{\varepsilon}=4 \times 10^{-3} \mathrm{~s}^{-1}$.

2.3 This figure compares the model isochronous curves at $T=550^{\circ} \mathrm{C}$ to the Section III, Division 5 isochronous curves for Grade 91 at the same temperature. In order from top to bottom the curves are the hot tensile curve and isochronous curves for 1, 10, 100, 1,000, 10,000, 100,000, and 300,000 hours. The model curves are plotted with solid lines, the Code curves with dashed lines. . . . . . . . . . . . . . . . . . .

2.4 Plot comparing the model values of yield stress to the Code values of $S_{y}$ for Grade 91 steel. To approximate the Code adjustment procedure used to calculate $S_{y}$ from material test data the actual model yield stress, as determined from simulated tension tests, is multiplied by 0.67 .

2.5 Design isochronous curves for the reference inelastic material model calculated from simulated creep tests beginning with as-received material. In order from top to bottom the curves are the hot tensile curve and isochronous curves for $1,10,100,1,000,10,000,100,000$, and 300,000 hours. . . . . . . . . . .

2.6 Model design fatigue curve at $T=540^{\circ} \mathrm{C}$ compared to the ASME Section III, Division 5 fatigue curve for Grade 91 steel at the same temperature. The plot also shows the simulated fatigue experiment data used to create the model curve along with the effect of applying the ASME factors of 2 on the (simulated) experimental strain range and 20 on the (simulated) experimental cycles to failure. . . . . . . . . . . . . . . . . .

2.7 Larson-Miller rupture correlation used to generate design values of the rupture stress $S_{r}$ for consistent comparisons to the inelastic model. . . . . . . . . .

2.8 The model damage diagram compared to the ASME Section III, Division 5 diagram for Grade 91 steel. The figure also shows the results of the simulated creep-fatigue experiments used to create the model diagram. . . . . . . . . .

3.1 Sketch illustrating the reference component used to assess the EPP strain limits design method. . . . . . . . . . . . . . . . . . .

3.2 Predicted design life comparison between the unmodified EPP ratcheting strain design procedure and the reference inelastic model. . . . . . . . . . .

3.3 Example comparing the softened isochronous curves to the reference curves using creep data from as-received material. This example shows unsoftened and softened curves for $T=550^{\circ} \mathrm{C}$. The curves in order from top to bottom are isochronous curves for 1, 10, 100, 1,000, 10,000, 100,000, and 300,000 hours. The presoftened curves were collected from simulated creep data where the material was first precycled with 1000 cycles of fully reversed strain-controlled loading with $\Delta \varepsilon=0.004$ at a strain rate of $10^{-3} \mathrm{~s}^{-1}$. 
3.4 Softened isochronous curves for the reference inelastic material model. These curves are constructed from simulated creep data after the material is precycled with 100 cycles of fully reversed, strain controlled loading at $\Delta \varepsilon=0.004$. In order from top to bottom the curves are isochronous curves for 1, 10, 100, $1,000,10,000,100,000$, and 300,000 hours. . . . . . . . . . . . .

3.5 Softened isochronous curves for the reference inelastic material model. These curves are constructed from simulated creep data after the material is precycled with 250 cycles of fully reversed, strain controlled loading at $\Delta \varepsilon=0.004$. In order from top to bottom the curves are isochronous curves for 1, 10, 100, $1,000,10,000,100,000$, and 300,000 hours. . . . . . . . . . . .

3.6 Softened isochronous curves for the reference inelastic material model. These curves are constructed from simulated creep data after the material is precycled with 500 cycles of fully reversed, strain controlled loading at $\Delta \varepsilon=0.004$. In order from top to bottom the curves are isochronous curves for 1, 10, 100, $1,000,10,000,100,000$, and 300,000 hours. . . . . . . . . . . . .

3.7 Softened isochronous curves for the reference inelastic material model. These curves are constructed from simulated creep data after the material is precycled with 1000 cycles of fully reversed, strain controlled loading at $\Delta \varepsilon=0.004$. In order from top to bottom the curves are isochronous curves for 1, 10, 100, $1,000,10,000,100,000$, and 300,000 hours. . . . . . . . . . . . .

3.8 Plot comparing the predicted design lives from the reference inelastic model to the lives predicted by the unmodified EPP strain limits procedure using unsoftened isochronous curves and the modified procedure using the softened curves. . . . . . . . . . . . . . . . . . . .

3.9 Softening factors for $T=550^{\circ} \mathrm{C}$ plotted as a function of design life and number of precycles. . . . . . . . . . . . . . . .

3.10 Example showing how the model, with the damage model turned off, comes to a steady saturated flow stress under cyclic loading. The figure plots the maximum stress for each cycle of repeated strain controlled cyclic loading with $\Delta \varepsilon=0.0047$ at three different temperatures. . . . . . . . . . . . .

3.11 Experimental fatigue data (personal communication, M. Li, Argonne National Laboratory) for Grade 91 at $T=600^{\circ} \mathrm{C}$ overlaid with a hypothetical saturated state. . . . . . . . . . . . . . . . . .

4.1 Sketch illustrating the reference component used to assess the EPP creepfatigue design method. . . . . . . . . . . . . . . . .

4.2 Plot comparing the design lives predicted by the unmodified EPP creep-fatigue design procedure to the reference inelastic model. . . . . . . . . . . . .

5.1 Figure showing how the experimental data (solid lines) was extrapolated out to a saturated state (dashed lines). . . . . . . . . . . . . . 
Initial development and extension of EPP methods to Grade 91

July 2018

\section{List of Tables}

2.1 Flow model parameters. . . . . . . . . . . . . . . . . . . 7

2.2 Temperature dependent hardening/softening parameters. . . . . . . . . 8

2.3 Damage model parameters. . . . . . . . . . . . . . . . 10

5.1 Table showing the recommended, model-based, and, where available experimental isochronous curve softening factors for Grade 91. . . . . . . . . . . . 35

5.2 Table showing the recommended softening factors for $S_{y}$ for Grade $91 . \quad$. . 36 



\section{Introduction}

Nuclear Code Cases N-861 and N-862 establish design by elastic perfectly plastic (EPP) analysis rules to cover the ASME Section III, Division 5 [5] ratcheting strain accumulation and creep-fatigue damage design limits [3, 4]. The current Code Cases only allow the use of the EPP rules for the Class A austenitic stainless steels $304 \mathrm{H}$ and $316 \mathrm{H}$.

The EPP methods have several advantages over the traditional Division 5 design by elastic analysis and design by inelastic analysis rules. The EPP methods still use a simplified structural analysis, like the elastic provisions, but do not require complicated modifications to the resulting stress and strain fields to account for creep and plasticity. The EPP rules are relatively easy to execute and can serve as screening tools for potential structural designs.

The purpose of this report is to extend the EPP rules to cover $9 \mathrm{Cr}-1 \mathrm{Mo}-\mathrm{V}$ (Grade 91) ferritic-martensitic steel. The primary difference in the structural response of the austenitic steels compared to Grade 91 is that the austenitic steels are cyclic hardening whereas Grade 91 is cyclic softening at elevated temperatures [14, 23, 24]. Under repeated cyclic load the flow stress of the austenitic steels tends to increase, whereas for Grade 91 it tends to decrease. This softening is caused by the meta-stable dislocation substructure found in Grade 91 in the as-received condition. Even at moderately high temperatures this dislocation structure immediately begins to undergo recovery, leading to a reduction in the dislocation density and the flow stress.

A previous report on this topic [21] noted that the bounding theorems underlying the EPP method for ratcheting strain accumulation preclude a cyclic softening material response [1, 2, 7-9]. However, that report also notes that a modified version of the EPP method could be used for a material with a saturating softening response. The cyclic flow stress in this type of material decreases initially with cycling but eventually reaches a cyclic steady state where the flow stress remains constant. This idea of a saturated softened state is critical to the modified EPP methods proposed here.

The approach taken in this report is to compare the EPP design methods to consistent full inelastic simulations. The reference inelastic model approximates the key features of the response of Grade 91 steel - specifically cyclic softening and creep-fatigue failure. Capturing this last mechanism requires adding a damage model to the standard model used to represent cyclic viscoplasticity in the material [10-13].

This comparison can be applied to assess both the ratcheting strain accumulation and the creep-fatigue design methods. The idea is to first generate consistent design data from simulated virtual experiments using the reference model. This ensures that a comparison between the inelastic model and the design method is fair - the data used in the design calculation matches the reference model.

The results of the comparisons show that the EPP strain limits design method requires modifications to conservatively bound the response of softening materials like Grade 91 . This report describes the theory underlying the proposed modifications and describes how to alter the existing Code Case to make the appropriate changes. The inelastic comparisons show that the EPP creep-fatigue method does not require any modification to apply to Grade 91. As described in detail in the report, the D-diagram used to represent creep-fatigue interaction in the EPP method already captures the effects of cyclic softening on creep-fatigue life. This report then describes two complete EPP methods for Grade 91 steel and demonstrates the conservatism of the proposed methods when compared to full inelastic simulations. 
Chapter 2 describes the consistent comparison method in detail and provides the reference inelastic model used in the remainder of the report. Chapter 3 presents the results of the consistent comparisons for the EPP strain limits methods and describes modifications to the methodology required to account for the cyclic softening response of Grade 91. The next chapter describes a similar comparison for the EPP creep-fatigue method. Here, however, the conclusion is that no modifications to the existing EPP Code Case is required. Chapter 5 describes the changes to the existing Code Cases that will be required to implement the methods for Grade 91 steel. The final chapter summarizes the report's conclusions. 


\section{Verifying design methods using full inelastic analysis}

\subsection{Verification strategy}

The approach adopted here to test the existing EPP Code Cases, develop modifications for softening materials, and test those modifications is a comparison to a full inelastic analysis. For these comparisons we define a reference inelastic model. The goal of the design method being tested then is to appropriately bound the response of this reference model for some realistic structural component.

The key requirement of this approach is that the reference inelastic model describe a realistic material response. This includes material failure - a reference model used to assess a creep-fatigue design method must including a damage model that realistically describes material failure under cyclic creep-fatigue loading. This is a difference between the type of inelastic model required here and the type of inelastic model used for Section III, Division 5 design by inelastic analysis. A material model used for Code design does not require, and arguably should not include, a damage model. However, a reference model for assessing a design method must fail realistically.

The model does not need to perfectly describe the response of an existing HBB Class A material. Instead, its response simply must be representative of the types of metallic material used in high temperature nuclear components. This greatly reduces the amount of work required to create the reference model as gathering experimental data and calibrating a model is often a long process.

Making a fair verification comparison between the reference model and the design method under consideration requires consistent design data. Simplified design methods often rely on tables of representative or bounding, depending on the design method, material properties. When used for a verification comparison this design data should be consistent with the reference inelastic model. The easiest way to create this consistent design data is to follow the same practices used to generate design tables for an actual material from experimental data, but instead of physical experiments use corresponding virtual experiments, i.e. simulations of experimental tests using the reference inelastic model.

This approach of verifying a design method by comparison to a reference model has several advantages over a comparison to either standard lab experimental tests or actual component failure data. In general, this approach eliminates most of the uncertainties in material response, loading conditions, and structural geometry inherent in comparing to actual test data. There is no variance in the response of the inelastic model - it will describe the same constitutive response each time it is invoked, requiring no bounds on heat-toheat variation, differences in product form, etc. Similarly, because the reference data are calculated with numerical simulations the loading conditions and component geometries are known exactly, eliminating the need to bound this source of uncertainty. In contrast to simplified lab experiments, finite element analysis allows consistent comparisons to be made for realistic structural components subjected to realistic high temperature loads.

However, ultimately this method of design procedure verification must be viewed as providing a necessary, but not sufficient, test. Fundamentally, this verification strategy tests the mechanical bounding theorems underlying a design method. Many design methods have their origin in theoretical work deriving a bound on some relevant design quantity (deformation, stress, creep-fatigue damage, etc.) assuming some particular material response. These 
bounding theorems are then generalized to practical design methods.

The consistent comparison method can be used to probe the limits of the bounding method, for example to determine which types of material response the method can safely bound. This is exactly the type of test required for the present work, which aims to assess whether an existing or modified EPP method can bound creep-fatigue damage and strain accumulation in a softening material like Grade 91.

However, a complete design method must also account for the uncertainties described above: variations in material constitutive response and applied loading, deviations in the as-built geometry, etc. These requirements would necessitate altering the theoretical bound, for example by incorporating lower bound, rather than average, material properties or by including load and/or resistance factors in the design method. A comparison to a reference inelastic model cannot directly assess the adequacy of a method for addressing these uncertainties.

That said, this method can serve to uncover potential flaws in design methodologies. If a design method fails to pass this type of check, that is if it fails to adequately bound the response of a some simulated structure with a known, reference inelastic constitutive response, it demonstrates that there is a fundamental flaw in the theory underlying the method. A design method that fails such a check is unlikely to be successful in engineering practice.

\subsection{Reference material model}

The first task in assessing the EPP methods with a consistent comparison to full inelastic simulations is to create the reference inelastic model. The key material feature of interest is cyclic softening and so the reference model must replicate a realistic softening material response. Beyond that, as discussed above, the model does not need to exactly reproduce the response of any of the existing Class A materials. However, as the purpose of this report is to establish the applicability of the EPP methods to the design of Grade 91 structures, the model developed here closely matches the average constitutive response of Grade 91 as represented by the design data contained in Section III, Division 5.

\subsubsection{Constitutive model}

\subsubsection{Base model for inelastic deformation}

The reference inelastic model used here builds on a model for material rate sensitivity described in a previous report [22]. The model accounts for a change in the character of inelastic deformation from rate insensitive at lower temperatures and faster strain rates to rate sensitive at higher temperatures and slower rates. Fundamentally, the model uses a unified viscoplastic decomposition of the total strain rate into elastic, inelastic, and thermal strain parts

$$
\dot{\varepsilon}=\dot{\varepsilon}_{e}+\dot{\varepsilon}_{v p}+\dot{\varepsilon}_{t h}
$$

The thermal strain rate comes from the instantaneous coefficient of thermal expansion Grade 91 steel, as described in Section II of the ASME Code:

$$
\dot{\varepsilon}_{t h}=\alpha \dot{T} \mathbf{I}
$$


where $\alpha$ is the temperature-dependent thermal expansion coefficient, $\dot{T}$ is the temperature rate, and $\mathbf{I}$ is the identity tensor. The elastic component is proportional to the stress rate through the isotropic elasticity tensor defined by the temperature dependent Section II elastic constants for Grade 91

$$
\dot{\varepsilon}_{e}=\mathrm{C}^{-1} \cdot \dot{\boldsymbol{\sigma}} .
$$

The inelastic component switches between a rate independent and a rate dependent update depending on the current value of the Kocks-Mecking normalized activation energy [16, 17, 20]

$$
g=\frac{k T}{\mu b^{3}} \log \frac{\dot{\varepsilon}_{0}}{\dot{\varepsilon}}
$$

where $k$ is the Boltzmann constant, $T$ absolute temperature, $\mu$ the temperature dependent shear modulus (as implied by the Section II temperature dependent Young's modulus and Poisson's ratio), $b$ the Burgers vector, $\dot{\varepsilon}_{0}$ a reference strain rate, and $\dot{\varepsilon}$ the current strain rate. A critical value of the activation energy, $g_{0}$ defines the switch point:

$$
\dot{\varepsilon}_{v p}= \begin{cases}\dot{\varepsilon}_{r i} & g \leq g_{0} \\ \dot{\varepsilon}_{r d} & g>g_{0} .\end{cases}
$$

The rate independent update is

$$
\begin{array}{rlr}
\dot{\boldsymbol{\varepsilon}}_{r i} & = & \gamma \frac{\partial f}{\partial \boldsymbol{\sigma}} \\
\gamma & \geq & 0 \\
f\left(\boldsymbol{\sigma}, \boldsymbol{h}, \sigma_{0}\right) & \leq & 0 \\
\gamma f\left(\boldsymbol{\sigma}, \boldsymbol{h}, \sigma_{0}\right) & = & 0 \\
\gamma \dot{f}\left(\boldsymbol{\sigma}, \boldsymbol{h}, \sigma_{0}\right) & = & 0
\end{array}
$$

and the rate dependent update is

$$
\begin{array}{rr}
\dot{\boldsymbol{\varepsilon}}_{r d}= & \gamma \frac{\partial f}{\partial \boldsymbol{\sigma}} \\
\gamma= & \left\langle\frac{f^{n}}{\eta}\right\rangle .
\end{array}
$$

Here, $f$ is some flow function or yield surface, for the rate-dependent and rate independent updates, respectively, and $\mathbf{h}$ are the model history variables. One key aspect of this model is that the rate-dependent and rate-independent updates consistently use the same $f$ and the same $\mathbf{h}$ so that prior history in the rate-independent regime affects subsequent ratedependent deformation and vice-versa.

For the reference model the flow parameters are, for the rate-independent update

$$
\sigma_{0}=\mu e^{C}
$$




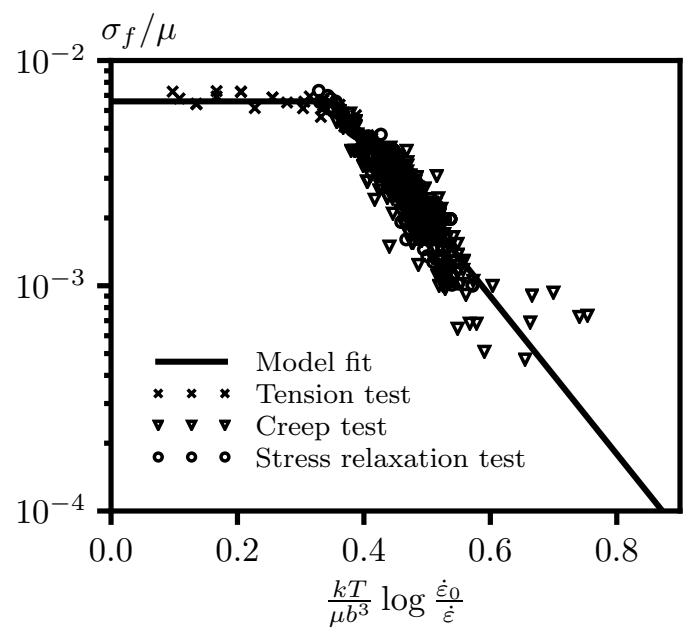

Figure 2.1: Kocks-Mecking master curve for Grade 91 steel, as established from a large experimental database.

and

$$
\begin{array}{cr}
\sigma_{0}= & 0 \\
n= & -\frac{\mu b^{3}}{k T A} \\
\eta= & e^{B} \mu \dot{\varepsilon}_{0}^{k T A /\left(\mu b^{3}\right)}
\end{array}
$$

for the rate-dependent update.

The flow constants $A, B, C$, and $g_{0}$ are fit using a Kocks-Mecking diagram. The reference material model uses the Kocks-Mecking diagram for Grade 91 steel and so it has, at least approximately, the same flow characteristics. Figure 2.1 shows the Kocks-Mecking diagram for Grade 91, as defined by a large database of experiments. These diagrams plot the flow stress, normalized by the temperature-dependent shear modulus, versus the Kocks-Mecking activation energy. More details on how to create these diagrams and extract the relevant material properties are provided in [22].

From this data $C$ is the ordinate value of the horizontal line describing the rate-insensitive normalized flow stress. $A$ is the slope and $B$ the intercept of the linear portion of the curve describing the material's rate sensitive flow stress. $g_{0}$ is the cutoff activation energy separating the two regimes.

The reference material model uses a standard $J_{2}$ flow rule and yield surface

$$
f=J_{2}(\boldsymbol{\sigma}-\boldsymbol{x})-\sqrt{\frac{2}{3}} \sigma_{i}
$$

The Kocks-Mecking model gives the base material flow stress. A hardening/softening model completes the description of the material in an undamaged state. Here this model represents a material that undergoes cyclic and work softening. The basic hardening model is a Chaboche model $[12,13]$, including the static recovery terms, supplemented with a 


\begin{tabular}{cc}
\hline Parameter & Value \\
\hline$A$ & -8.06 \\
$B$ & -2.18 \\
$C$ & -5.02 \\
$g_{0}$ & 0.349 \\
$\dot{\varepsilon}_{0}$ & $10^{10} \mathrm{~s}^{-1}$ \\
$b$ & $2.48 \times 10^{-7} \mathrm{~mm}$ \\
\hline
\end{tabular}

Table 2.1: Flow model parameters.

Voce model for isotropic softening. This model has three history variables, the scalar $\dot{\bar{\varepsilon}}_{v p}$ equivalent inelastic strain with evolution equation

$$
\dot{\bar{\varepsilon}}_{v p}=\sqrt{\frac{2}{3} \dot{\varepsilon}_{v p}: \dot{\varepsilon}_{v p}}
$$

and two backstresses $\mathbf{x}_{1}$ and $\mathbf{x}_{2}$, each with the evolution equation

$$
\dot{\mathbf{x}}_{i}=\left(\frac{2}{3} C_{i} \mathbf{n}-\sqrt{\frac{2}{3}} \gamma_{i}\left(\bar{\varepsilon}_{v p}\right) \mathbf{x}_{i}\right) \dot{\gamma}-A_{i} \sqrt{\frac{3}{2}}\left\|\mathbf{x}_{i}\right\|^{a_{i}-1} \mathbf{x}_{i} .
$$

The isotropic term in Eq. 2.17 is given by the Voce equation

$$
\sigma_{i}=\sigma_{0}+Q\left(1-\exp \left(-\delta \bar{\varepsilon}_{v p}\right)\right)
$$

and the total backstress is the sum of the two individual backstresses

$$
\mathbf{x}=\sum_{i=1}^{n} \mathbf{x}_{i}
$$

These equations fully define the undamaged response of the reference inelastic model. The Kocks-Mecking flow parameters are temperature independent as the dependence of flow stress with temperature is a part of the Kocks-Mecking model for thermal activation. Table 2.1 gives these temperature independent material properties. In general the parameters defining the models for the flow hardening/softening model are temperature dependent. For the reference model these parameters are described at five temperature control points. The model linearly interpolates the parameters to values of temperature in between the five control temperatures. Table 2.2 lists the temperature-dependent model parameters. As mentioned above, the model elastic and thermal properties are take directly from the properties of Grade 91 steel described in Section II of the ASME Code.

This form is fairly typical for high temperature modeling of structural steels. Note however that the Voce saturation stress $Q$ is negative. This isotropic softening competes with the kinematic hardening to make the model work hardening but cyclic softening at low temperatures and both work and cyclic softening at elevated temperatures. This matches the experimentally-observed response of Grade 91. 


\begin{tabular}{cccccc}
\hline Parameter & $25^{\circ} \mathrm{C}$ & $500^{\circ} \mathrm{C}$ & $550^{\circ} \mathrm{C}$ & $600^{\circ} \mathrm{C}$ & $650^{\circ} \mathrm{C}$ \\
\hline$Q$ & $-100 \mathrm{MPa}$ & $-80.0 \mathrm{MPa}$ & $-100 \mathrm{MPa}$ & $-100 \mathrm{MPa}$ & $-130 \mathrm{MPa}$ \\
$\delta$ & 2.0 & 1.61 & 0.810 & 2.0 & 4.5 \\
$C_{1}$ & $27000 \mathrm{MPa}$ & $40100 \mathrm{MPa}$ & $27500 \mathrm{MPa}$ & $27500 \mathrm{MPa}$ & $27500 \mathrm{MPa}$ \\
$\gamma_{1}$ & 350 & 360 & 300 & 350 & 350 \\
$A_{1}$ & $1.38 \times 10^{-15}$ & $6.87 \times 10^{-16}$ & $1.27 \times 10^{-15}$ & $1.00 \times 10^{-15}$ & $8.43 \times 10^{-16}$ \\
$a_{2}$ & 3.32 & 2.69 & 4.19 & 4.04 & 5.92 \\
$C_{2}$ & $7000 \mathrm{MPa}$ & $0 \mathrm{MPa}$ & $0 \mathrm{MPa}$ & $0 \mathrm{MPa}$ & $0 \mathrm{MPa}$ \\
$\gamma_{2}$ & 92 & 0 & 0 & 0 & 0 \\
$A_{1}$ & $2.32 \times 10^{-16}$ & 0 & 0 & 0 & 0 \\
$a_{2}$ & 3.45 & 0 & 0 & 0 & 0 \\
\hline
\end{tabular}

Table 2.2: Temperature dependent hardening/softening parameters.

\subsubsection{Damage model}

This model for inelastic deformation must be supplemented with a model for creep-fatigue damage. The goal here is to represent, as much as possible, the failure behavior of Grade 91 steel to creep-fatigue, pure creep, and low cycle fatigue loading. Additionally, the model must interface cleanly with an existing model for deformation. This last requirement is an implementation concern: the damage model was implemented as a subsequent addition to the already-implemented model for deformation described in the previous section. Additionally, the damage models were implemented in a generic framework so that several different damage formulations could be examined before arriving at the final model described below.

The damage formulation starts with a base model for material deformation $\boldsymbol{\sigma}\left(\boldsymbol{\varepsilon}_{n+1}, \boldsymbol{h}_{n+1}\right)$. In this context, this is the deformation model described in the previous subsection. The damage model supplements this base model with a model for the evolution of a scalar damage model, which in turn modifies the stress update:

$$
\begin{array}{ll}
\boldsymbol{\sigma}_{n+1}= & \left(1-\omega_{n+1}\right) \boldsymbol{\sigma}\left(\boldsymbol{\varepsilon}_{n+1}, \boldsymbol{h}_{n+1}\right) \\
\omega_{n+1}= & w\left(\varepsilon_{n+1}, \frac{\boldsymbol{\sigma}_{n+1}}{1-\omega_{n+1}}, \omega_{n+1}\right) .
\end{array}
$$

Given this definition of a scalar damage model as supplementing an existing deformation model, there are two implementation challenges. The first is to solve the modified, damaged stress update given the next step total strain $\varepsilon_{n+1}$ and the material history and the second is to modify the material model's algorithmic tangent to be consistent with the superimposed damage model.

The modified stress update solves the implicit integration of the stress evolution equation and the scalar damage evolution equation:

$$
\left[\begin{array}{l}
\boldsymbol{R}_{1} \\
R_{2}
\end{array}\right]=\left[\begin{array}{c}
\boldsymbol{\sigma}_{n+1}-\left[1-\omega_{n+1}\right] \boldsymbol{\sigma}\left(\boldsymbol{\varepsilon}_{n+1}, \boldsymbol{h}_{n+1}\right) \\
\omega_{n+1}-w\left(\boldsymbol{\varepsilon}_{n+1}, \frac{\boldsymbol{\sigma}_{n+1}}{1-\omega_{n+1}}, \omega_{n+1}\right)
\end{array}\right]=\left[\begin{array}{l}
\mathbf{0} \\
0
\end{array}\right]
$$

where the left side of this equation is a residual to be zeroed. The implementation solves 
this equation with Newton's method, requiring the Jacobian of the residual equation

$$
\boldsymbol{J}=\left[\begin{array}{cc}
\boldsymbol{I} & \boldsymbol{\sigma}\left(\varepsilon_{n+1}, \frac{\boldsymbol{\sigma}_{n+1}}{1-\omega_{n+1}}, \boldsymbol{h}_{n+1}\right) \\
-\frac{\partial \omega}{\partial \boldsymbol{\sigma}_{n+1}} \frac{1}{1-\omega_{n+1}} & 1-\frac{\partial \omega}{\partial \omega_{n+1}}-\frac{1}{\left(1-\omega_{n+1}\right)^{2}} \frac{\partial \omega}{\partial \boldsymbol{\sigma}_{n+1}}: \boldsymbol{\sigma}_{n+1}
\end{array}\right] .
$$

The damage model must provide, in addition to the update equation $w$, the derivatives $\frac{\partial w}{\partial \sigma}$ and $\frac{\partial w}{\partial \omega}$. Note each iteration computing the residual and its Jacobian calls the base material stress update $\boldsymbol{\sigma}\left(\varepsilon_{n+1}, \boldsymbol{h}_{n+1}\right)$, which itself typically involves solving a nonlinear systems of equations using an iterative scheme. So this method of superimposing damage on an existing deformation model saves time in implementation but may be computationally more costly than directly solving the coupled damage and deformation update equations.

This modified, damaged stress update alters the definition of the material algorithmic tangent

$$
\mathbf{A}_{n+1}=\frac{d \boldsymbol{\sigma}_{n+1}}{d \boldsymbol{\varepsilon}_{n+1}} .
$$

The modified tangent is

$$
\boldsymbol{A}_{n+1}=\left(1-\omega_{n+1}\right) \boldsymbol{A}_{n+1}^{\prime}-\boldsymbol{\sigma}_{n+1}^{\prime} \otimes \frac{d \omega_{n+1}}{d \boldsymbol{\varepsilon}_{n+1}}
$$

with the primed (') quantities representing the base material update and algorithmic tangent, without damage. Given this base material model the only new derivative to be determined is $\frac{d \omega_{n+1}}{d \varepsilon_{n+1}}$.

Using the implicit function theorem

$$
\begin{gathered}
\frac{d \omega}{d \boldsymbol{\varepsilon}}=\frac{\partial \omega}{\partial \boldsymbol{\varepsilon}}+\left(\frac{\partial \omega}{\partial \boldsymbol{\sigma}^{\prime}} \cdot \frac{\partial \boldsymbol{\sigma}^{\prime}}{\partial \omega}\right) \frac{d \omega}{d \boldsymbol{\varepsilon}}+\frac{\partial \omega}{\partial \boldsymbol{\sigma}^{\prime}} \cdot \frac{\partial \boldsymbol{\sigma}^{\prime}}{\partial \boldsymbol{\sigma}} \frac{d \boldsymbol{\sigma}}{d \boldsymbol{\varepsilon}}+\frac{\partial \omega}{\partial \omega} \frac{d \omega}{d \boldsymbol{\varepsilon}} \\
\frac{d \omega}{d \boldsymbol{\varepsilon}}=\frac{\partial \omega}{\partial \boldsymbol{\varepsilon}}+\left(\frac{1}{1-\omega} \frac{\partial \omega}{\partial \boldsymbol{\sigma}^{\prime}} \cdot \boldsymbol{\sigma}^{\prime}\right) \frac{d \omega}{d \boldsymbol{\varepsilon}}+\frac{1}{1-\omega} \frac{\partial \omega}{\partial \boldsymbol{\sigma}^{\prime}} \cdot \frac{d \boldsymbol{\sigma}}{d \boldsymbol{\varepsilon}}+\frac{\partial \omega}{\partial \omega} \frac{d \omega}{d \boldsymbol{\varepsilon}} \\
\left(1-\frac{1}{1-\omega} \frac{\partial \omega}{\partial \boldsymbol{\sigma}^{\prime}} \cdot \boldsymbol{\sigma}^{\prime}-\frac{\partial \omega}{\partial \omega}\right) \frac{d \omega}{d \boldsymbol{\varepsilon}}=\frac{\partial \omega}{\partial \boldsymbol{\varepsilon}}+\frac{1}{1-\omega} \frac{\partial \omega}{\partial \boldsymbol{\sigma}^{\prime}} \cdot \frac{d \boldsymbol{\sigma}}{d \boldsymbol{\varepsilon}}
\end{gathered}
$$

Let $k=1-\frac{1}{1-\omega} \frac{\partial \omega}{\partial \boldsymbol{\sigma}^{\prime}} \cdot \boldsymbol{\sigma}^{\prime}-\frac{\partial \omega}{\partial \omega}$ then

$$
\frac{d \omega}{d \boldsymbol{\varepsilon}}=\frac{1}{k} \frac{\partial \omega}{\partial \boldsymbol{\varepsilon}}+\frac{1}{k(1-\omega)} \frac{\partial \omega}{\partial \boldsymbol{\sigma}^{\prime}} \cdot \frac{d \boldsymbol{\sigma}}{d \boldsymbol{\varepsilon}}
$$

Combined this with Eq. 2.27:

$$
\begin{aligned}
& \boldsymbol{A}_{n+1}=\left(1-\omega_{n+1}\right) \boldsymbol{A}_{n+1}^{\prime}-\frac{1}{k}\left(\boldsymbol{\sigma}_{n+1}^{\prime} \otimes \frac{\partial \omega}{\partial \boldsymbol{\varepsilon}}\right)-\frac{1}{k(1-\omega)}\left(\boldsymbol{\sigma}_{n+1}^{\prime} \otimes \frac{\partial \omega}{\partial \boldsymbol{\sigma}^{\prime}}\right) \cdot \boldsymbol{A}_{n+1} \\
& \boldsymbol{A}_{n+1}=\left(1-\omega_{n+1}\right) \boldsymbol{A}_{n+1}^{\prime}-\frac{1}{k}\left(\boldsymbol{\sigma}_{n+1}^{\prime} \otimes \frac{\partial \omega}{\partial \boldsymbol{\varepsilon}}\right)-\frac{1}{k(1-\omega)}\left(\boldsymbol{\sigma}_{n+1}^{\prime} \otimes \frac{\partial \omega}{\partial \boldsymbol{\sigma}^{\prime}}\right) \cdot \boldsymbol{A}_{n+1}
\end{aligned}
$$




\begin{tabular}{ll}
\hline Parameter & Value \\
\hline$C$ & 562.04 \\
$n$ & 0.0 \\
$m$ & 2.2675 \\
$c_{1}$ & 2.9574 \\
$c_{2}$ & 2.0 \\
$c_{3}$ & 0.01 \\
\hline
\end{tabular}

Table 2.3: Damage model parameters.

$$
\boldsymbol{A}_{n+1}=\left\{\boldsymbol{I}+\frac{1}{k(1-\omega)}\left(\boldsymbol{\sigma}_{n+1}^{\prime} \otimes \frac{\partial \omega}{\partial \boldsymbol{\sigma}^{\prime}}\right)\right\}^{-1}\left\{\left(1-\omega_{n+1}\right) \boldsymbol{A}_{n+1}^{\prime}-\frac{1}{k}\left(\boldsymbol{\sigma}_{n+1}^{\prime} \otimes \frac{\partial \omega}{\partial \boldsymbol{\varepsilon}}\right)\right\} .
$$

Equation 2.34 gives an expression for the new algorithmic tangent, a total derivative, using only partial derivatives of the damage model and the existing undamaged material algorithmic tangent. Two of the three partial derivatives of the damage function are required for the stress update Jacobian, described above. The third, $\frac{\partial \omega}{\partial \varepsilon}$ is unique to modified tangent calculation. All three must be provided along with the damage function.

The particular damage model used for the reference inelastic model uses the damage evolution rate

$$
\dot{\omega}=C \beta(\omega) \bar{\sigma}^{n} \dot{\bar{\varepsilon}}^{m}
$$

where $\bar{\sigma}$ is the standard von Mises equivalent stress and the $\beta$ function is defined as

$$
\beta(x)=\frac{\Gamma\left(c_{1}+c_{2}\right)}{\Gamma\left(c_{1}\right)+\Gamma\left(c_{2}\right)}\left(\left(c_{1}-1\right) x^{c_{1}-2}(x-1)^{c_{2}-1}-\left(c_{2}-1\right) x^{c_{1}-1}(1-x)^{c_{2}-2}\right)-c_{3}
$$

with $C, c_{1}, c_{2}$, and $c_{3}$ all parameters and $\Gamma$ the standard gamma function. The actual damage function $w$ is an implicit integration of this damage rate

$$
w=w_{n}+\dot{\omega} \Delta t_{n+1}
$$

Damage is then proportional to the effective stress and total effective strain rates raised to some power. The $\beta$ function accelerates damage at the end of life to achieve a realistic rapid failure over the last few load cycles and furthermore smoothly decreases the material stress to zero at the end of life to allow using the damaged material in finite element simulations. Essentially, the $\beta$ function smoothly kills an element in a finite element calculation, rather than allowing the stresses to continue into negative values or abruptly zeroing the element stresses both of which can lead to numerical convergence problems. The constants $c_{1}$ and $c_{2}$ calibrate this end of life behavior while constant $c_{3}$ sets the initial rate of damage accumulation.

Table 2.3 lists the damage model parameters used for the reference inelastic model. These parameters are temperature independent. They were calibrated to approximate the failure characteristics of Grade 91 at $550^{\circ} \mathrm{C}$. 


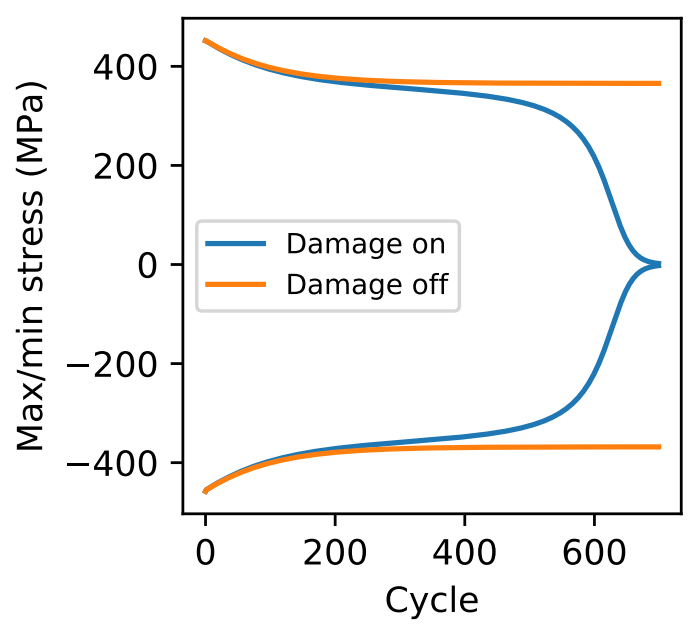

Figure 2.2: Example simulations of pure fatigue loading comparing the model with the damage model turned on and without damage. The loading conditions are $T=540^{\circ} \mathrm{C}$, $\Delta \varepsilon=0.01 \mathrm{~mm} / \mathrm{mm}$ full reversed loading, and $\dot{\varepsilon}=4 \times 10^{-3} \mathrm{~s}^{-1}$.

\subsubsection{Example response}

The equations and parameters described in the previous subsection fully defined the reference inelastic model. Figure 2.2 shows results from a simulation demonstrating the effect of the damage model. This is a material point simulations of fully-reversed fatigue loading. The plot shows two example calculations - one using the deformation model without the damage model and the second with the damage model turned on. The figure plots the minimum and maximum stresses over each load cycle as a function of cycle count. Without the damage model the material softens until it reaches some final, saturated material state. After this saturation point the cyclic flow stress becomes constant. With the damage model turned on rather than saturate the flow stress continues to decrease - at first slowly but serrating near the end of life. The $\beta$ function ensures that after abruptly falling off, effectively indicating the end of the material fatigue life, the stresses smoothly approach zero.

Figure 2.3 compares the model isochronous curves, generated by simulating creep tests, to the ASME Section III, Division 5 isochronous curves for Grade 91. Both the model and the Code curves are for $T=550^{\circ} \mathrm{C}$. This comparison shows that the model approximates the response of Grade 91 to creep loading, particularly for long hold times As described above, the goal of the reference inelastic model was not to exactly reproduce the average behavior of Grade 91 steel, but rather to reproduce the essential characteristic under study here - cyclic and work softening - while maintaining a realistic or representative response to other types of load. This figure shows the reference material model behaves reasonably for pure creep loading.

\subsubsection{Design data}

Consistently comparing this reference inelastic model to some design approach requires consistent design data that describes the reference model's response. The EPP strain limits 


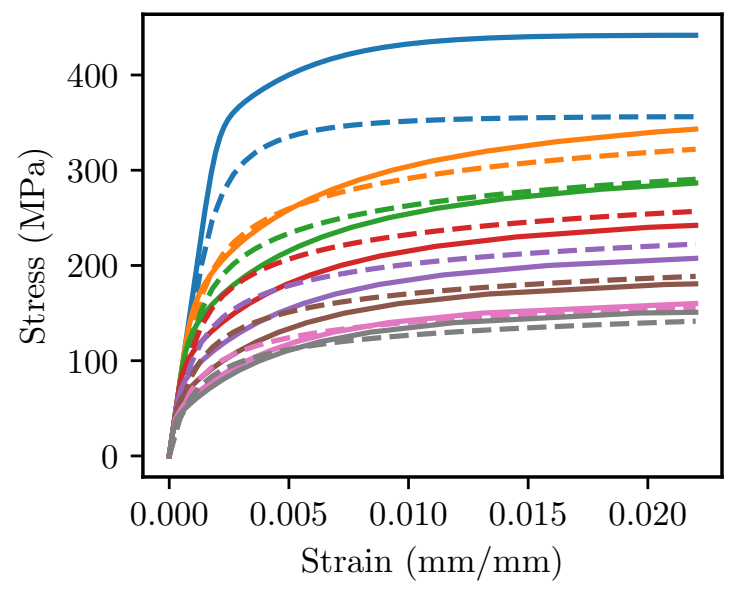

Figure 2.3: This figure compares the model isochronous curves at $T=550^{\circ} \mathrm{C}$ to the Section III, Division 5 isochronous curves for Grade 91 at the same temperature. In order from top to bottom the curves are the hot tensile curve and isochronous curves for 1, 10, 100, 1,000, 10,000, 100,000, and 300,000 hours. The model curves are plotted with solid lines, the Code curves with dashed lines.

Code Case requires values of the material yield stress $S_{y}$ and the material isochronous curves. The creep-fatigue Code Case requires fatigue curves, creep rupture curves, and a damage diagram. This subsection describes obtaining these required data using simulated experiments with the reference inelastic model and the standard ASME approach for converting the (simulated) experimental data into the design charts.

Figure 2.4 compares the values of $S_{y}$ obtained for the reference inelastic model to the Code values for Grade 91 steel. The ASME approach for determining $S_{y}$ is to first create a database of yield stress values at a standard strain rate, commonly the ASTM E-21 [6] value of $8.33 \times 10^{-5} \mathrm{~s}^{-1}$, and at different temperatures. A best-fit trend line is established for this baseline data. Then the trend line is uniformly scaled downwards so that the trend line value for the yield stress at room temperature matches the minimum room temperature yield stress for the material, as established by the relevant material standard.

To approximate this process for the deterministic reference model where there is no specification minimum yield stress we first simulated a large number of tensile tests at different temperatures and at the ASTM E-21 standard strain rate. A best-fit trend line can be drawn through this simulated experimental data. However, the reference model is deterministic - it does not represent stochastic heat-to-heat material variation nor does it account for different product forms. To approximate the ASME lower bound process we multiplied the best-fit trend line by a factor of 0.67. This value is often used in the ASME code to move from lower bound to average values of $S_{y}$. Indeed, as the figure shows, the scaled trend line does decently approximate the Code values of $S_{y}$ for Grade 91 . This scaled trend line is used to define the consistent values of $S_{y}$ for the EPP design comparisons in subsequent chapters of this report. A more comprehensive procedure would be use the Grade 91 minimum specified yield stress and follow the full ASME scaling procedure.

The ASME Section III isochronous curves are supposed to represent the average creep 


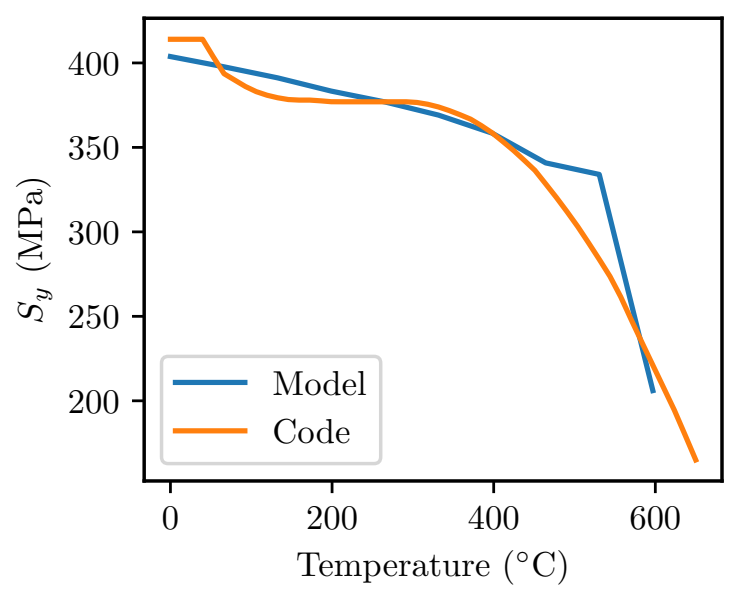

Figure 2.4: Plot comparing the model values of yield stress to the Code values of $S_{y}$ for Grade 91 steel. To approximate the Code adjustment procedure used to calculate $S_{y}$ from material test data the actual model yield stress, as determined from simulated tension tests, is multiplied by 0.67 .

response of the Class A materials. For consistent design curves we simulated a large series of creep tests at different temperatures and applied load levels. This creep data was directly converted into the design isochronous curves shown in Figure 2.5. No bounding procedure is required because the Code curves are supposed to represent average material behavior.

Section III of the ASME Code presents fatigue data as plots of number of cycles to failure versus strain range. The underlying experimental data is from strain controlled cyclic tests. To generate corresponding consistent data from the reference inelastic model we simulated a large series of strain controlled tests for different, fully reversed strain ranges and at $T=540^{\circ} \mathrm{C}$. Figure 2.6 plots the number of cycles to failure extracted from the simulation results. This failure data agrees with the ASME Code data for Grade 91 for a low number of cycles to failure. No effort was made to adjust the model to match the Code high cycle fatigue data as the reference model will not be used in this loading regime. The ASME Code fatigue curves apply two safety factors: a factor of 2 to the experimental strain range and a factor of 20 on the experimental cycles to failure. The design fatigue curve is the worst case of the two modified curves. The figure reproduces this approach and shows the resulting, bounding fatigue curve for the reference inelastic model.

The Code uses a Larson-Miller [19] correlation to creep-fatigue data to establish design rupture stresses $S_{r}$. The Larson-Miller parameter is

$$
L M=T\left(\log t_{r}+C\right)
$$

where $T$ is absolute temperature, $t_{r}$ is the experimental rupture life, and $C$ a constant, typically about 20 . The Code assumes that a material's creep rupture stress correlates to this parameter. This correlation is typically established through a Larson-Miller plots, which plot the logarithm of the rupture stress versus the Larson-Miller parameter for a large number of creep-rupture tests. Figure 2.7 reproduces this approach for the reference inelastic model. 


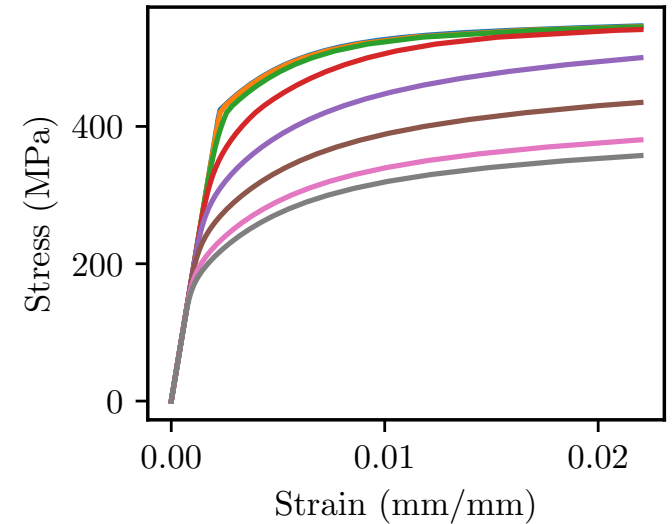

(a) $\mathrm{T}=400^{\circ} \mathrm{C}$

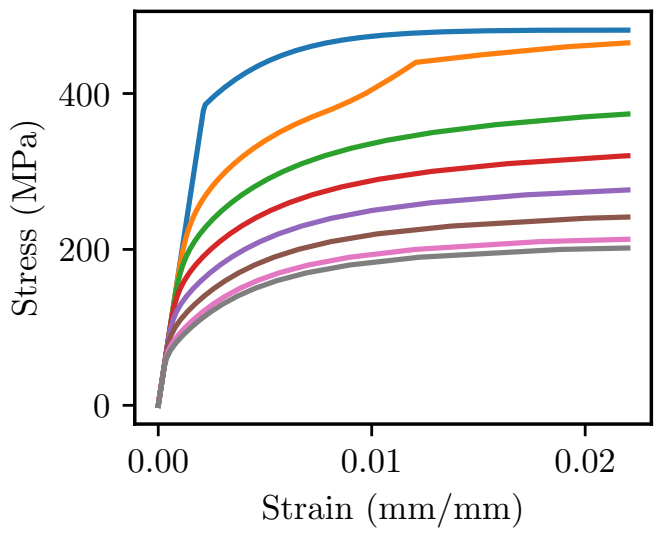

(c) $\mathrm{T}=500^{\circ} \mathrm{C}$

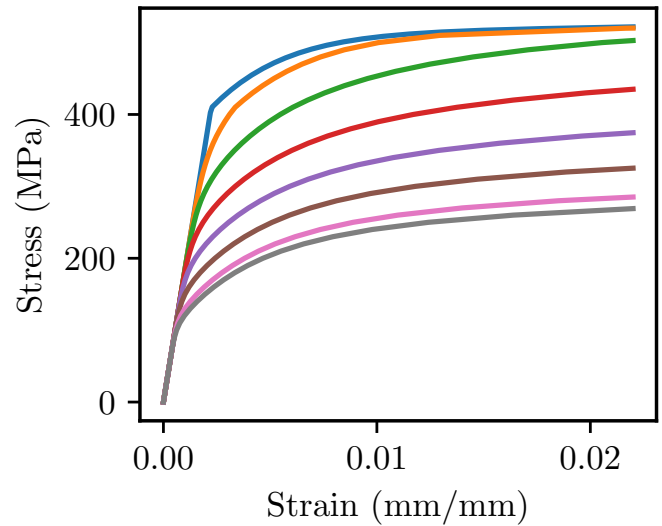

(b) $\mathrm{T}=450^{\circ} \mathrm{C}$

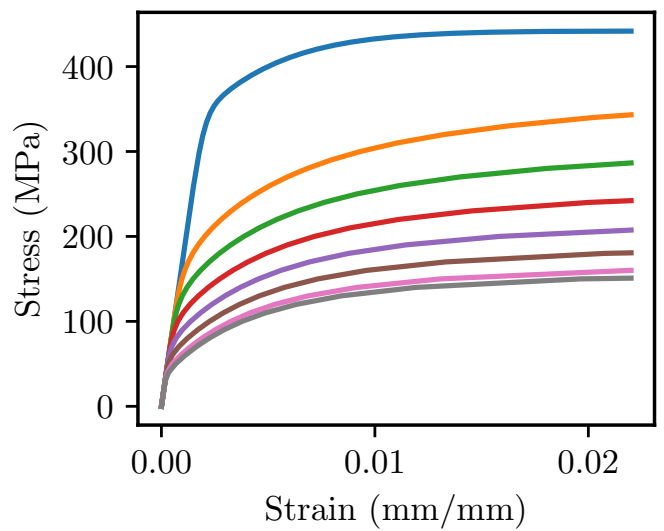

(d) $\mathrm{T}=550^{\circ} \mathrm{C}$

Figure 2.5: Design isochronous curves for the reference inelastic material model calculated from simulated creep tests beginning with as-received material. In order from top to bottom the curves are the hot tensile curve and isochronous curves for 1, 10, 100, 1,000, 10,000, 100,000 , and 300,000 hours. 


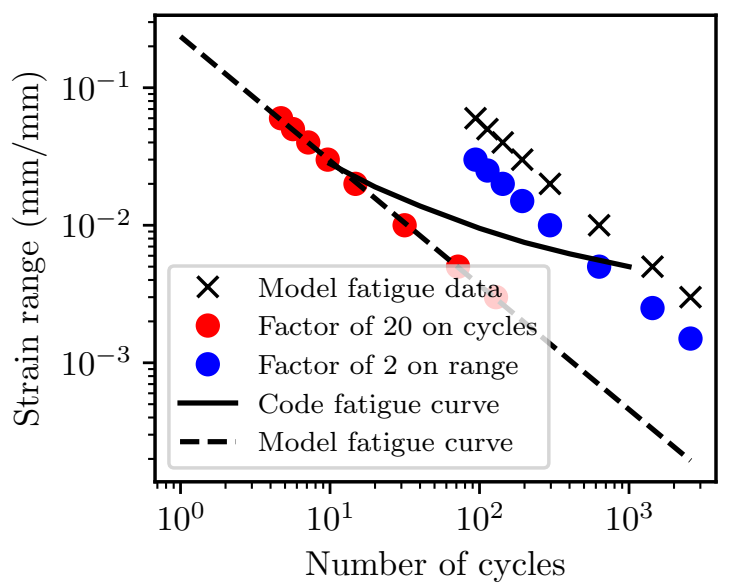

Figure 2.6: Model design fatigue curve at $T=540^{\circ} \mathrm{C}$ compared to the ASME Section III, Division 5 fatigue curve for Grade 91 steel at the same temperature. The plot also shows the simulated fatigue experiment data used to create the model curve along with the effect of applying the ASME factors of 2 on the (simulated) experimental strain range and 20 on the (simulated) experimental cycles to failure.

Here the experimental data comes from simulated rupture tests. The ASME approach is to determine a bounded lower bound fit to the experimental data.

The reference model produces deterministic rupture lives - for a given set of loading conditions the model predicts the same rupture time without any random variation. The scatter in the Larson Miller diagram comes from the process of simulating long-life creep rupture tests using the inelastic model. By necessity these simulations use a very large time step size, $\Delta t$. When the simulations predict failure the data indicates that the true simulated rupture life is somewhere between $t_{n}$ and $t_{n+1}=t_{n}+\Delta t$. Adaptive integration could be used to locate the rupture time more precisely, but this method was not applied here. As such, there is some scatter in the simulated rupture lives as the true time to rupture falls randomly in the final $\Delta t$ interval.

This small numerical variation does not replicate the relatively large experimental scatter in measured rupture lives. To approximate the ASME lower bound procedure the best-fit curve is simply shifted down by 10\%. A better approximation would be to transfer the actual measured scatter for Grade 91 rupture into the model. How, exactly, to accomplish this will be a topic for future work.

Finally, the EPP creep-fatigue procedure requires a damage diagram. This diagram is created using creep-fatigue experimental data. The procedure is, for each test:

1. Calculate the test fatigue damage $D_{f}=\frac{N}{N_{f}}$ where $N$ is the experimental number of cycles to failure and $N_{f}$ is the number of cycles to failure under pure fatigue loading predicted by the design fatigue curve without the factors of two and twenty.

2. Calculate the test creep damage computed as $D_{c}=\int \frac{d t}{t_{f}}$ where the integral domain is the entire test time history and $t_{f}$ is the time to failure predicted by the design 


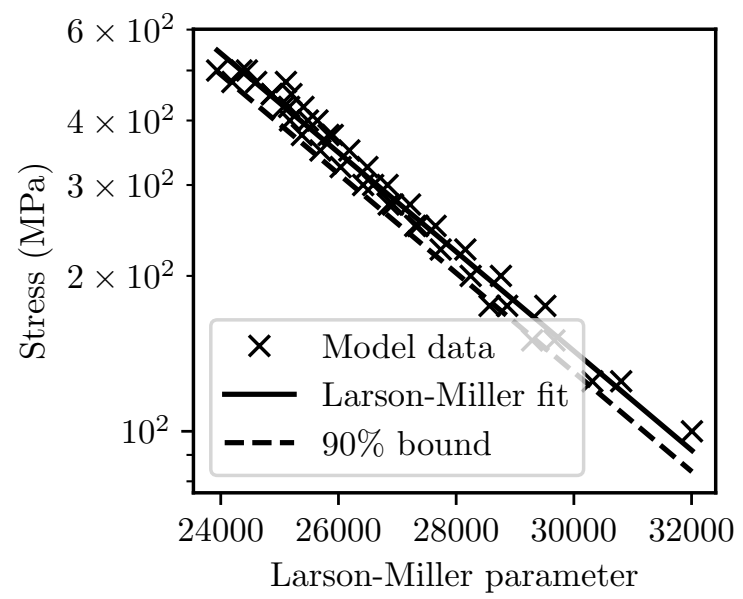

Figure 2.7: Larson-Miller rupture correlation used to generate design values of the rupture stress $S_{r}$ for consistent comparisons to the inelastic model.

creep-rupture correlation for average material behavior, not the lower bound.

3. Plot the $\left(D_{c}, D_{f}\right)$ point on the D-diagram.

This process is repeated for each test, which results in a scatter of points on a damagediagram like the one shown in 2.8. The final D-diagram is a design envelope describing the average behavior as typified by the experimental data. If a point falls inside the design envelope it represents a loading that passes the design creep-fatigue check. Conversely, points outside the envelope fail the design creep-fatigue check. Logically, the design envelope must pass through the points $(1,0)$ and $(0,1)$ on the diagram. Division 5 uses two straight-line segments to connect these points to some common intersection. This intersection point then fully defines the design envelope. This design envelope is selected based by approximating the plotted points representing creep-fatigue experiments. The envelope is supposed to represent the average material response. Bounds to accommodate material variation are applied to the design fatigue and creep-rupture curves, as described above.

Figure 2.8 shows the results of simulated creep-fatigue experiments with the reference inelastic model and the selected design envelope with intersection point $(0.075,0.01)$ overlaid on the Code design envelope for Grade 91 steel, which has an intersection of $(0.1,0.01)$. Qualitatively, the reference inelastic model behaves very similarly to Grade 91 steel. Both design D-diagrams predict severe creep-fatigue interactions.

The design information contained in Figs. 2.4 through 2.8 is sufficient to execute the EPP strain limits and creep-fatigue code cases. Design calculations using these material properties will be consistent with the reference inelastic model. 
Initial development and extension of EPP methods to Grade 91

July 2018

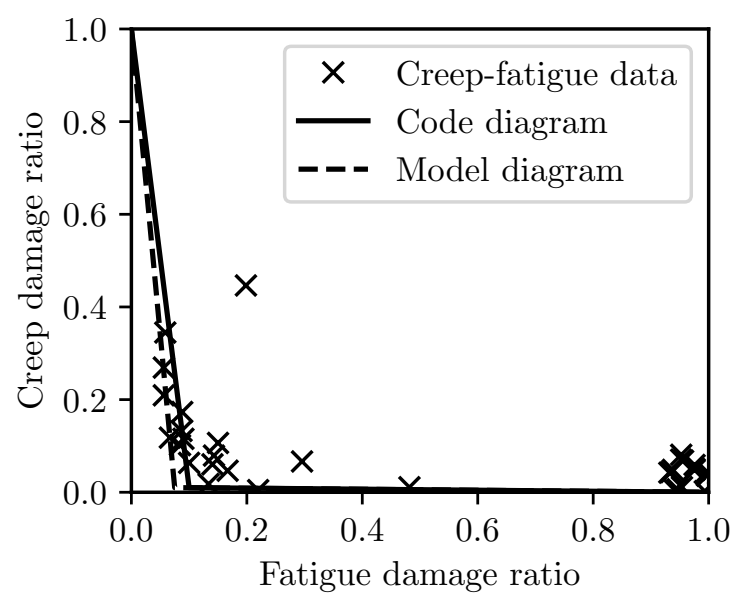

Figure 2.8: The model damage diagram compared to the ASME Section III, Division 5 diagram for Grade 91 steel. The figure also shows the results of the simulated creep-fatigue experiments used to create the model diagram. 



\section{Strain limits}

This chapter uses a consistent comparison to the full inelastic model, described in the previous section, to assess the conservatism of the existing EPP ratcheting strain accumulation rules, designed for use with cyclic hardening austenitic steel, when used with Grade 91. The conclusion is that the base rules are not conservative due to cyclic softening. We then propose a modified design procedure and demonstrate that this modified procedure is conservative when compared to full inelastic simulations.

The conclusions developed here agree with a previous report on the topic [21]. However, the calculations presented here are new and based on the full inelastic model described in the previous section.

\subsection{Unmodified Code Case}

Figure 3.1 shows the reference geometry used to compare the full inelastic model to the EPP design calculations. The model represents a section of a thin-walled pressure vessel away from geometric discontinuities like heads or nozzles. The vessel is subjected to a cyclic, linear temperature gradient where the outer wall temperature remains fixed at $250^{\circ} \mathrm{C}$ and the inner wall temperature cycles between $250^{\circ} \mathrm{C}$ and $550^{\circ} \mathrm{C}$. The heating and cooling rates are both $100^{\circ} \mathrm{C} /$ hour. At the same time, the vessel is under a constant pressure inducing a hoop stress of $\sigma_{\theta \theta}=110 \mathrm{MPa}$.

The analysis considers two types of cycles. The first $n_{\text {pre }}$ cycles have zero hold time. These impose some prior fatigue loading on the vessel. The remaining thermal cycles hold the vessel in the hot condition for 100 hours each cycle.

The EPP strain limits Code case requires creating a composite cycle that represents the key features of all the design loads. The EPP bounding analysis is rate independent and so hold times can be discounted when constructing the composite cycle. Figure 3.1 also shows the composite cycle corresponding to the full cycle. It simply removes the hold time from the full load history.

Two methods were used to determine the ratcheting design life of this structure. The first was a full inelastic analysis using the reference material model described in Chapter 2 , not including the damage model. These simulations first apply $n_{\text {pre }}$ cycles of preloading followed by repeating the full thermal cycle until the accumulated inelastic strains over the vessel wall exceed the ASME criteria:

1. Average strain of $1 \%$

2. Linearized maximum strain of $2 \%$

3. Maximum (peak) strain of $5 \%$.

The final number of cycles, $n_{\text {total }}$, was recorded along with the total time to failure.

A corresponding EPP calculation was used to determine the structure's design life. This calculation followed the rules contained in the Code Case for austenitic stainless steel, without modification [3]. This involves computing a pseudoyield stress used to bound deformation in the structure. This pseudoyield is based on the values of the material isochronous stressstrain curves and the material yield stress $S_{y}$. The base Code Case uses the ASME Section 

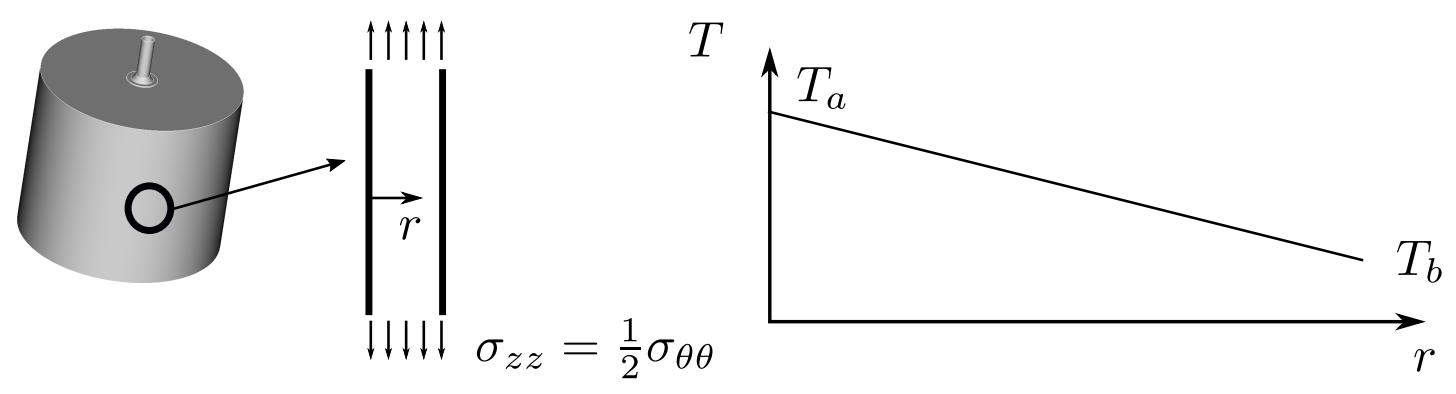

Linear through-wall thermal gradient

Full load cycle

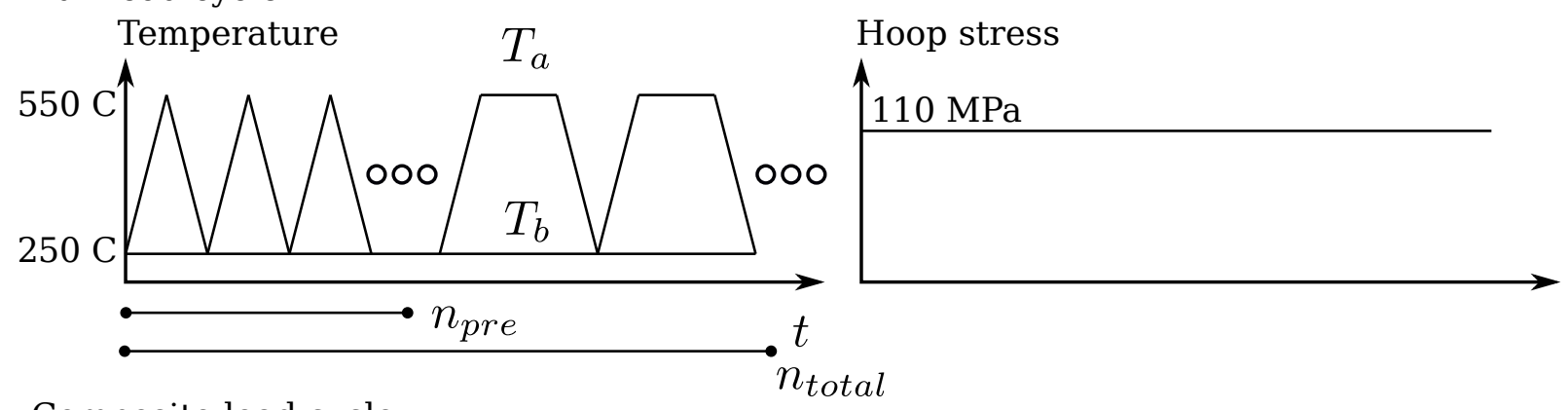

Composite load cycle

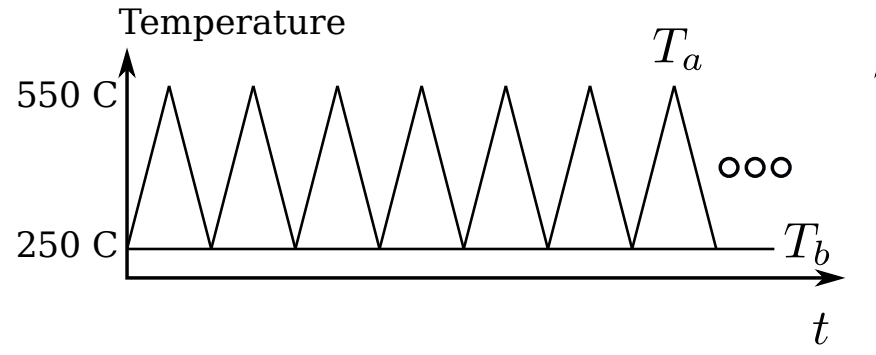

Hoop stress

$110 \mathrm{MPa}$

Figure 3.1: Sketch illustrating the reference component used to assess the EPP strain limits design method. 


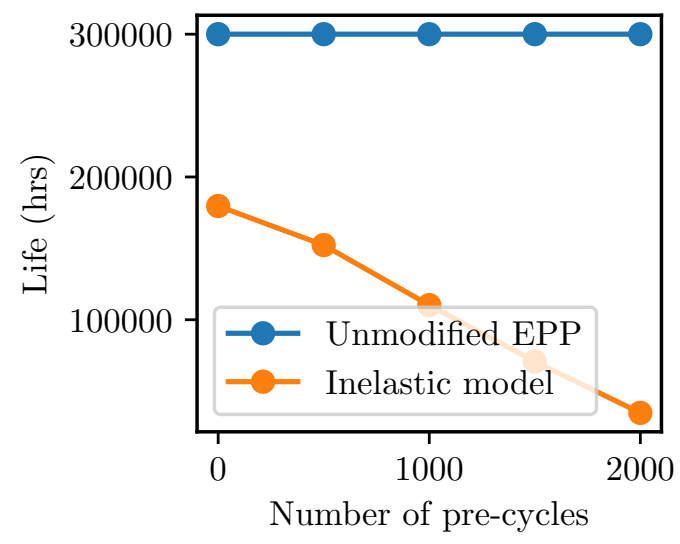

Figure 3.2: Predicted design life comparison between the unmodified EPP ratcheting strain design procedure and the reference inelastic model.

III, Division 5 isochronous curves, which are based on material in the as-received condition - i.e. without any prior cycling.

The consistent EPP calculation uses the values of $S_{y}$ and the isochronous stress-strain curves established for the reference inelastic material model, without damage, in the previous chapter. This is the direct equivalent of following the existing EPP Code Case rules by using standard, non-cycled isochronous curves.

Figure 3.2 plots the ratcheting life of the structure as calculated with the inelastic model versus the EPP design ratcheting life. Both lives are plotted as function of of the number of prior fatigue cycles. The base EPP procedure produces non-conservative results - the EPP design life is greater than the actual time to $1 \%$ strain predicted by the inelastic model. The EPP design life is also constant no matter the number of pre-cycles. This is because the EPP composite load cycle for this problem is exactly the same for the pre-cycling and the main thermal cycling. Therefore, as the loading and bounding material properties are always the same, the Code Case procedure always returns the same design life.

The pre-cycling applied in this problem softens the material and increases the subsequent ratcheting deformation, leading to a shorter design life for the pre-cycled components. The existing EPP design method does not capture this effect. This consistent comparison then shows that modifications will be required to alter the EPP method to account for cyclic softening material behavior.

\subsection{Modified Code Case}

The EPP Code Case does not account for the effect of cyclic softening on subsequent creep deformation. For softening materials, like Grade 91, prior cycling increases the rate of subsequent creep leading to an increased ratcheting rate and a lower design life.

The Code Case only uses two types of material data to bound ratcheting strain accumulation: the material yield stress $S_{y}$ and the values of the isochronous stress-strain curves. Both of these properties will be affected by prior cyclic deformation in softening materials. 


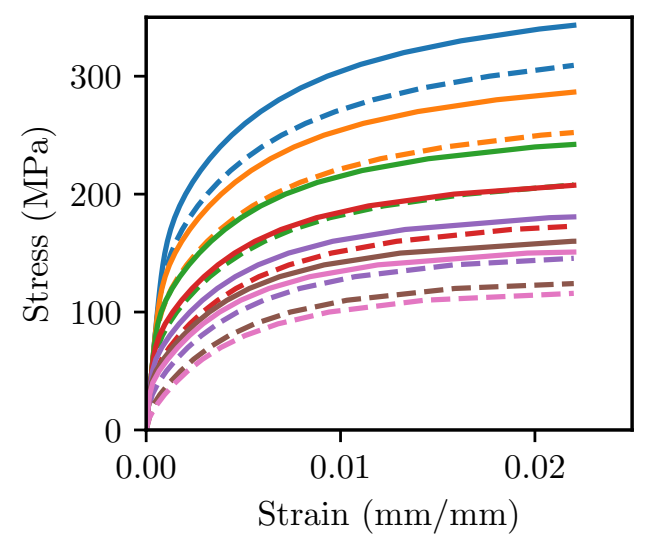

Figure 3.3: Example comparing the softened isochronous curves to the reference curves using creep data from as-received material. This example shows unsoftened and softened curves for $T=550^{\circ} \mathrm{C}$. The curves in order from top to bottom are isochronous curves for 1,10 , 100, 1,000, 10,000, 100,000, and 300,000 hours. The presoftened curves were collected from simulated creep data where the material was first precycled with 1000 cycles of fully reversed strain-controlled loading with $\Delta \varepsilon=0.004$ at a strain rate of $10^{-3} \mathrm{~s}^{-1}$.

Cycling will decrease the yield stress of the material and reduce the values of the isochronous stress-strain curve (by increasing the rate of subsequent creep deformation).

We can generate pre-softened isochronous curves and pre-softened values of the yield stress for the reference material model. The idea is simply to first simulate $n$ cycles of fully-reversed strain controlled fatigue loading at some $\Delta \varepsilon$, unload the simulation, and then simulate a uniaxial tension or creep test. For the creep data, a large collection of such pre-softened tests can be collected into a set of softened isochronous curves. Figure 3.3 shows an example of these softened isochronous stress-strain curves, comparing the softened curves to the unsoftened curves calculated in the previous chapter. As expected, prior cyclic deformation reduces the values of the curves.

This process can be repeated for a range of precycle counts $(n)$ and fully-reversed strain ranges $(\Delta \varepsilon)$. Figures 3.4-3.7 shows such softened curves for $400,450,500$, and $550^{\circ} \mathrm{C}$ and for $100,250,500$, and 1000 cycles of prior fatigue loading. The general trend is that increasing the number of pre-cycles decreases the values of the isochronous stress-strain curves. Similar simulated pre-cycled uniaxial tension tests established values of the softened material yield stress $S_{y}$.

We can now posit a modified EPP strain limits procedure that accounts for cyclic softening. The idea is to replace the material yield stress $S_{y}$ with the softened material yield stress and the values of the isochronous curves with their corresponding softened counterparts.

Figure 3.8 compares the design lives predicted by the full inelastic model, the unmodified EPP procedure, and the modified EPP procedure. The modified EPP procedure, accounting for cyclic softening, now bounds the lives predicted by the full inelastic model. This modified EPP strain limits procedure can then form the basis of an ASME Code Case allowing the use of the EPP ratcheting strain design procedure for Grade 91 steel. 


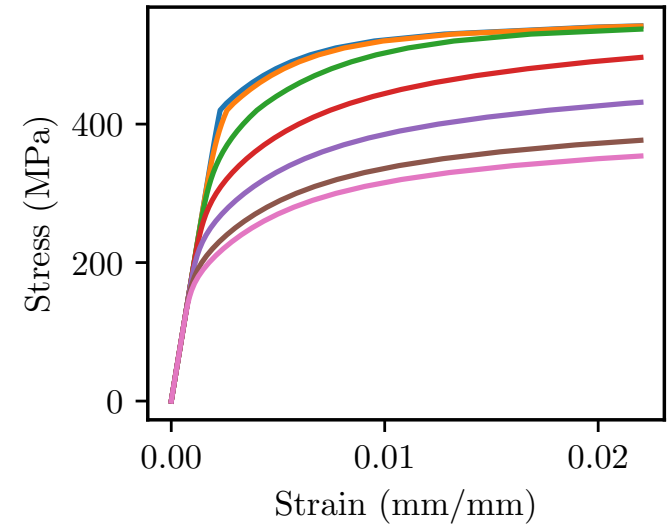

(a) $\mathrm{T}=400^{\circ} \mathrm{C}$

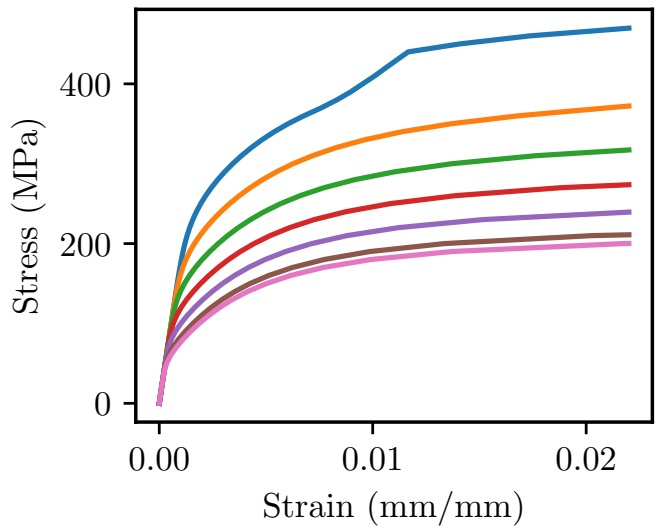

(c) $\mathrm{T}=500^{\circ} \mathrm{C}$

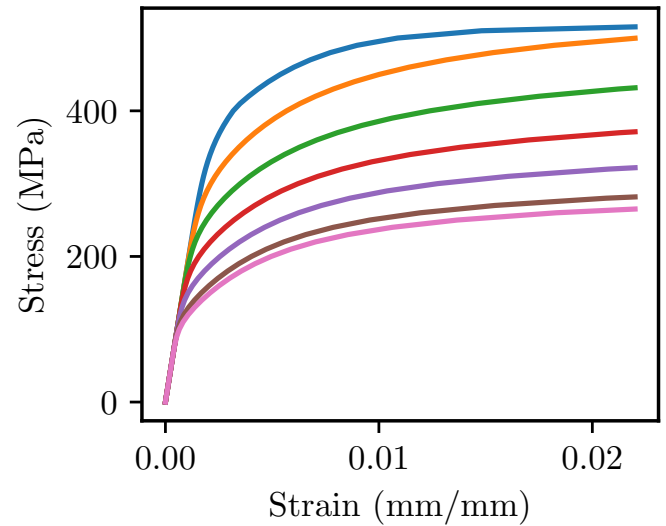

(b) $\mathrm{T}=450^{\circ} \mathrm{C}$

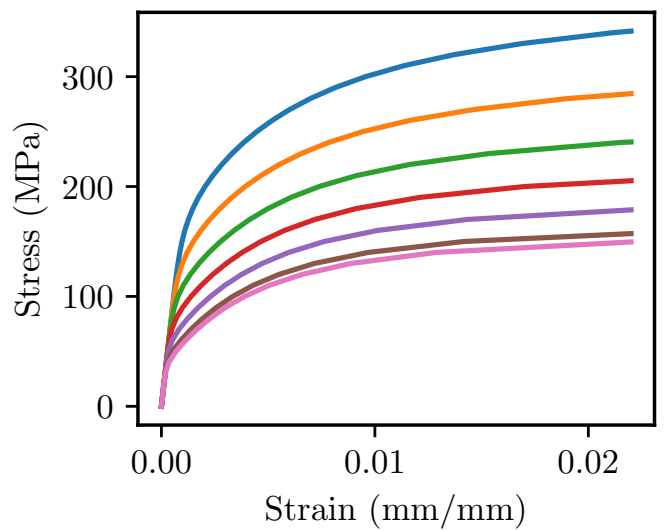

(d) $\mathrm{T}=550^{\circ} \mathrm{C}$

Figure 3.4: Softened isochronous curves for the reference inelastic material model. These curves are constructed from simulated creep data after the material is precycled with 100 cycles of fully reversed, strain controlled loading at $\Delta \varepsilon=0.004$. In order from top to bottom the curves are isochronous curves for 1, 10, 100, 1,000, 10,000, 100,000, and 300,000 hours. 


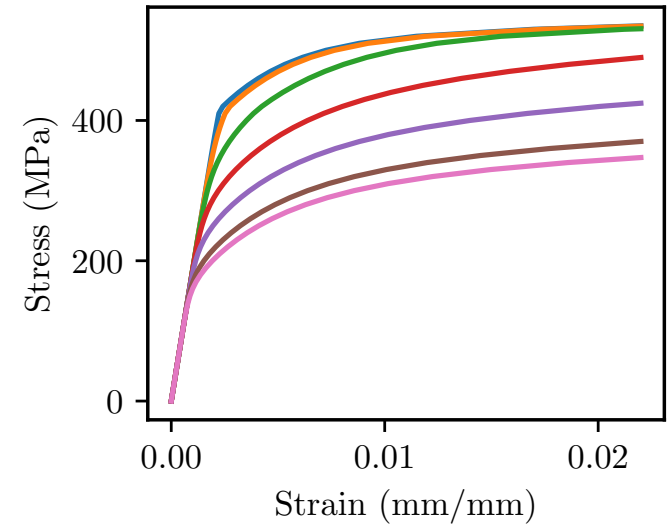

(a) $\mathrm{T}=400^{\circ} \mathrm{C}$

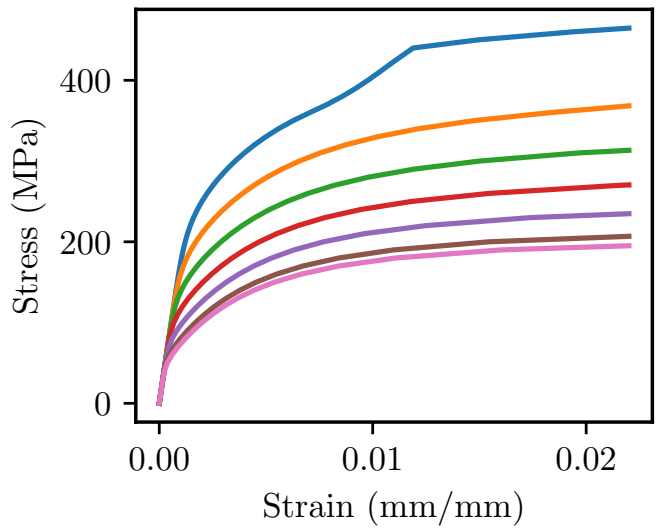

(c) $\mathrm{T}=500^{\circ} \mathrm{C}$

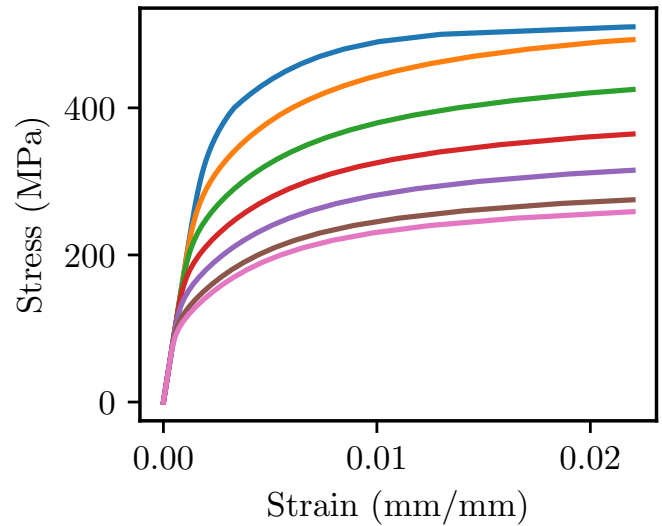

(b) $\mathrm{T}=450^{\circ} \mathrm{C}$

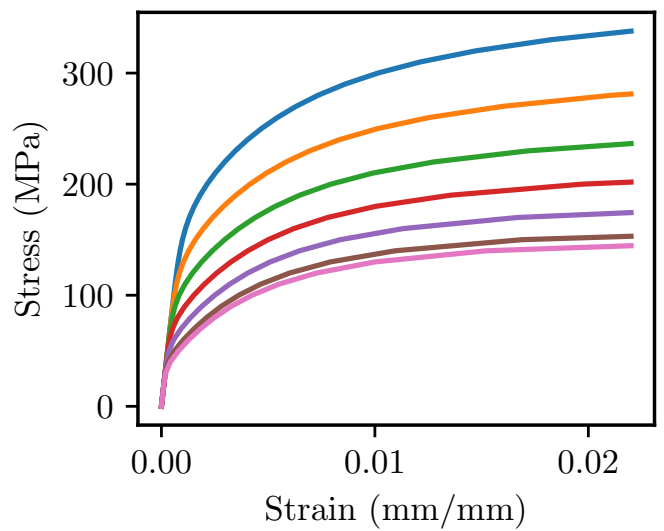

(d) $\mathrm{T}=550^{\circ} \mathrm{C}$

Figure 3.5: Softened isochronous curves for the reference inelastic material model. These curves are constructed from simulated creep data after the material is precycled with 250 cycles of fully reversed, strain controlled loading at $\Delta \varepsilon=0.004$. In order from top to bottom the curves are isochronous curves for 1, 10, 100, 1,000, 10,000, 100,000, and 300,000 hours. 


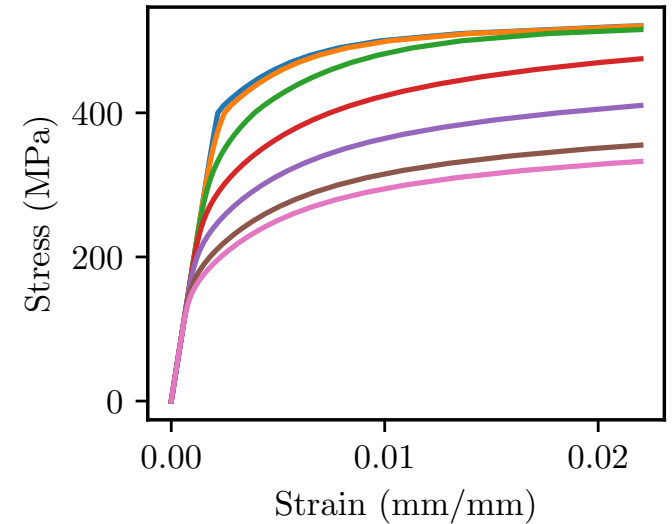

(a) $\mathrm{T}=400^{\circ} \mathrm{C}$

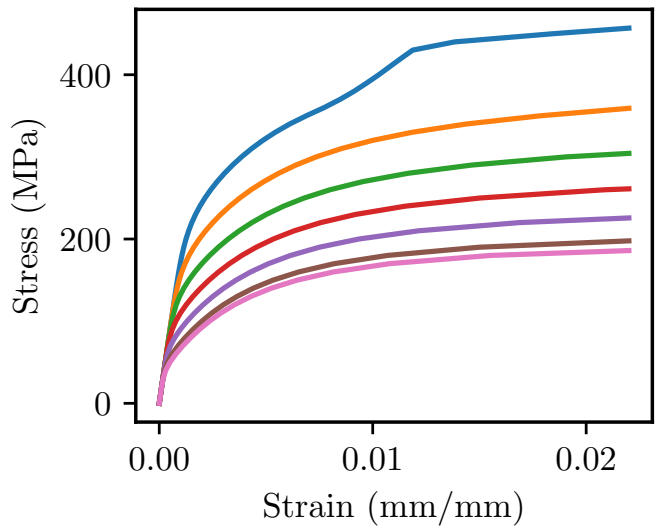

(c) $\mathrm{T}=500^{\circ} \mathrm{C}$

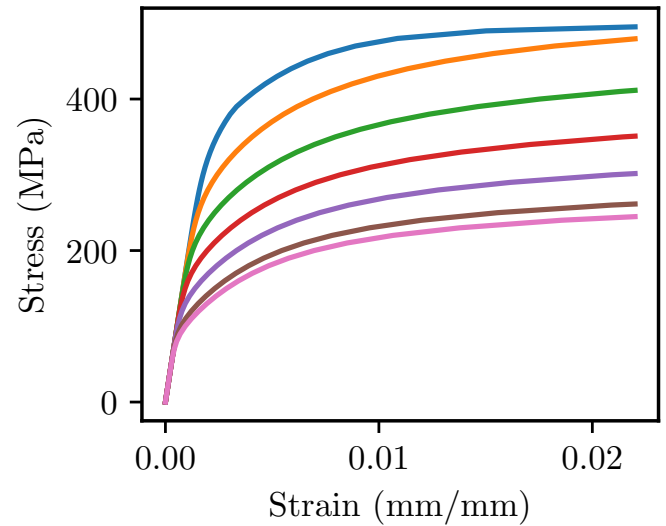

(b) $\mathrm{T}=450^{\circ} \mathrm{C}$

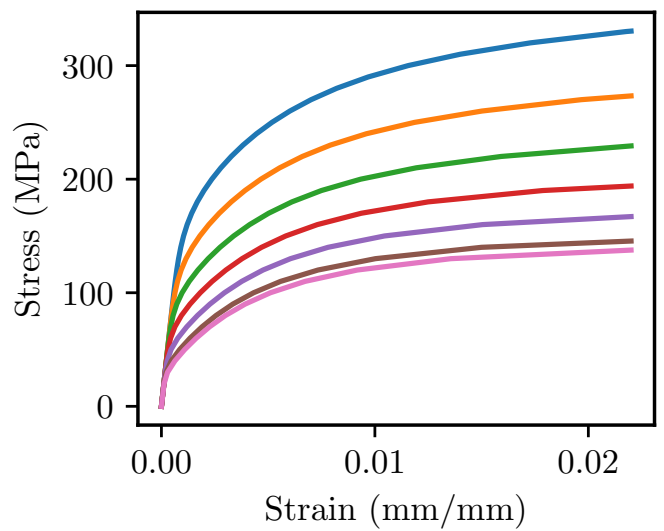

(d) $\mathrm{T}=550^{\circ} \mathrm{C}$

Figure 3.6: Softened isochronous curves for the reference inelastic material model. These curves are constructed from simulated creep data after the material is precycled with 500 cycles of fully reversed, strain controlled loading at $\Delta \varepsilon=0.004$. In order from top to bottom the curves are isochronous curves for 1, 10, 100, 1,000, 10,000, 100,000, and 300,000 hours. 


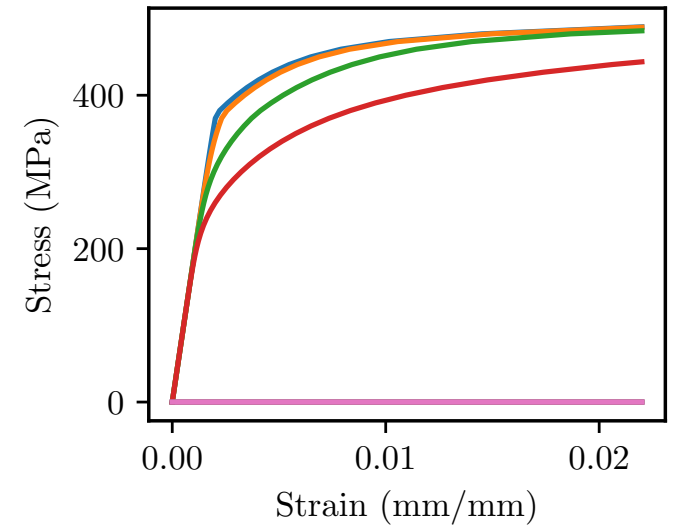

(a) $\mathrm{T}=400^{\circ} \mathrm{C}$

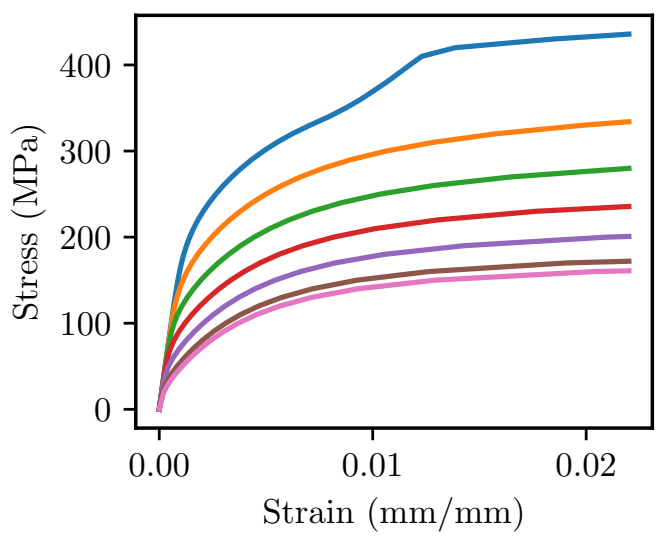

(c) $\mathrm{T}=500^{\circ} \mathrm{C}$

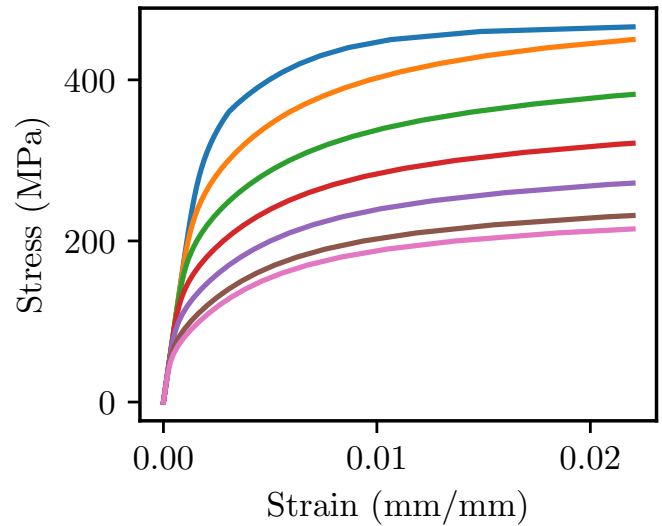

(b) $\mathrm{T}=450^{\circ} \mathrm{C}$

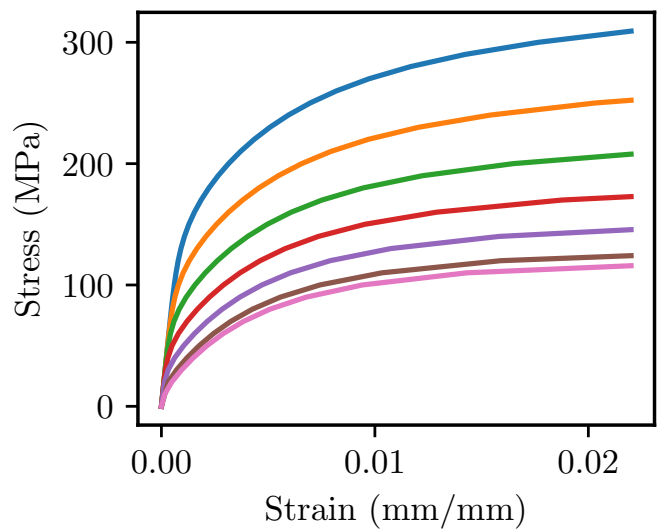

(d) $\mathrm{T}=550^{\circ} \mathrm{C}$

Figure 3.7: Softened isochronous curves for the reference inelastic material model. These curves are constructed from simulated creep data after the material is precycled with 1000 cycles of fully reversed, strain controlled loading at $\Delta \varepsilon=0.004$. In order from top to bottom the curves are isochronous curves for 1, 10, 100, 1,000, 10,000, 100,000, and 300,000 hours. 


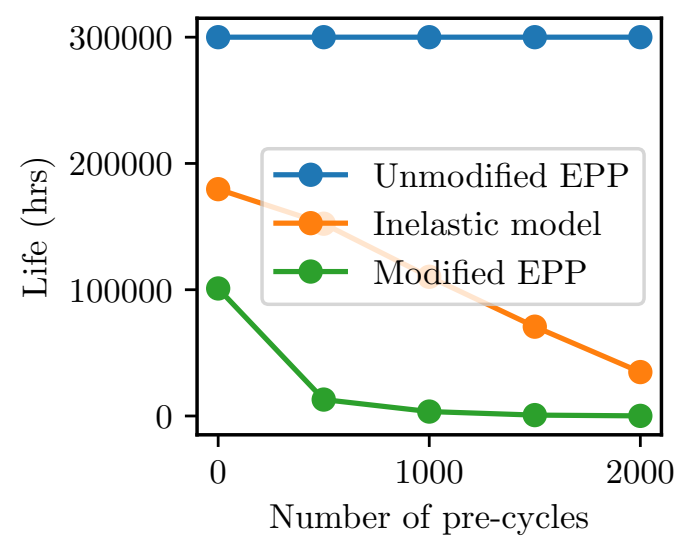

Figure 3.8: Plot comparing the predicted design lives from the reference inelastic model to the lives predicted by the unmodified EPP strain limits procedure using unsoftened isochronous curves and the modified procedure using the softened curves.

\subsection{Isochronous curve softening factors}

The main obstacle towards applying this modified procedure to Grade 91 is obtaining values of the softening material yield stress and softened isochronous curves. Conceptually, the values of the softened isochronous stress/strain curves are functions of:

1. Temperature $(T)$

2. Number of prior fatigue cycles $(n)$

3. Strain range in fatigue $(\Delta \varepsilon)$

4. Design life $(t)$

5. Strain $(\varepsilon)$.

Generating softened isochronous stress-strain curves for any combination of these parameters is straightforward when using the reference inelastic model. Experimentally it would be much more difficult to establish a suitable database of material properties. Additionally, in design practice some of this information may not be available either. For example, the existing EPP methods do not explicitly track the number of design cycles, but instead bound strain accumulation over a design life. Furthermore, there will be additional design issues, for example combining cycles of different amplitudes and at different temperature into a single measure of cyclic softening.

To start, consider the softened isochronous stress strain curves as some fixed reduction factor multiplied by the unsoftened isochronous curves collected from creep tests on material in the as-received condition. A table of the values of this reduction factor, as a function of the six variables listed above, could define the softened isochronous curves starting from the existing Section III, Division 5 curves in a future Code Case. 


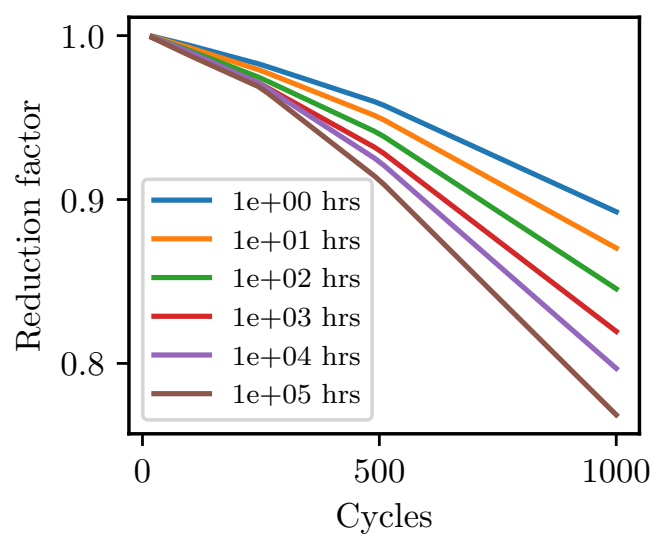

Figure 3.9: Softening factors for $T=550^{\circ} \mathrm{C}$ plotted as a function of design life and number of precycles.

This does not immediately reduce the number of variables required to define the softened isochronous curves. It is reasonable to assume that softening factors compiled for fully-reversed loading bound other loading ratios. Strain can be eliminated from the list of variables by noting that

1. The EPP Code Case only requires values of the isochronous stress-strain curves for inelastic strains less than $1 \%$.

2. The softening factors tend to increase as the accumulated strain increases.

Based on these assumptions, the softening factors are based on the isochronous curve values at $1 \%$ inelastic strain. These values should bound the values at the lower strain levels that could be used in executing the Code Case procedure.

Figure 3.9 plots the reduction ratio

$$
\frac{\sigma_{u s}\left(\varepsilon_{\text {inelastic }}=1 \%\right)}{\sigma_{s}\left(\varepsilon_{\text {inelastic }}=1 \%\right)}
$$

comparing the values of the isochronous stress-strain curve at $1 \%$ strain, at $550^{\circ} \mathrm{C}$, and for a fixed, fully-reversed strain range as a function of cycle count and for different design lives. In general, for the full inelastic model including damage, the softening factors do not saturate to some limiting value.

However, if the simulations are run with the damage model turned off the softened flow stress eventually saturates. Figure 3.10 illustrates this for simulated fatigue tests at different conditions. The number of cycles required to reach saturation varies with the applied strain range and the number of cycles, but eventually all the simulations reach this cyclic steady state. If the material reaches a cyclic steady state then the isochronous stress/strain curve reduction factors will likewise saturate. The value of the reduction factor in the steady-state condition bounds the reduction factor corresponding to any other combination of strain range or number of applied cycles. That is, the material will eventually reach this state 


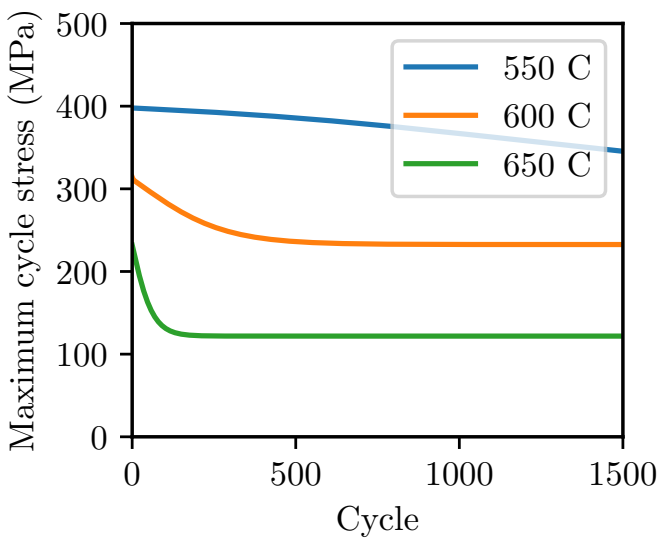

Figure 3.10: Example showing how the model, with the damage model turned off, comes to a steady saturated flow stress under cyclic loading. The figure plots the maximum stress for each cycle of repeated strain controlled cyclic loading with $\Delta \varepsilon=0.0047$ at three different temperatures.

regardless of the specific applied cyclic, strain controlled load. This, thus far hypothetical, cyclic steady state allows us to eliminate both the number of cycles and the strain range from the softening factors. This leaves only the temperature and the design life. The design life can be eliminated by considering the softening factor for the maximum allowable Code design life - currently 300,000 hours for Grade 91 but likely to be extended to 500,000 hours in the near future. This report considers both maximum design lives.

The final reduction factors would be a function of temperature only. This existing EPP Code Case could be easily modified to account for this softening factor.

However, does the actual material achieve some cyclic steady state? Figure 3.11 shows a sample strain-controlled fatigue tests on Grade 91 at $T=600^{\circ} \mathrm{C}$. The experimental results show that the material flow stress does not saturate but rather continues to decrease.

A closer look at the data shows two separate sources of softening. The maximum cycle stress decreases quickly at first but then the rate of change per cycle slows down as the material appears to trend towards some cyclic steady state. However, towards the end of the test the rate of changes increases again and the cyclic flow stress tends towards zero. This second trend is caused by the accumulation of fatigue damage in the material. This damage further softens the material, ultimately causing failure.

The cause of this damage induced softening is physically distinct from the initial cyclic softening. The initial softening is caused by dislocation recovery in the material reducing the sessile dislocation density and therefore reducing the flow stress. This process would eventually saturate if the material continued to deform under the same conditions.

In contrast, damage is caused by the nucleation, growth, and coalescence of voids in the material. This process is distinct from the recovery mechanism causing the initial cyclic softening and does not saturate.

Figure 3.11 illustrates an extrapolated saturated state assuming exponential decay following the initial portion of the softening curve and extrapolating out to the saturated flow 


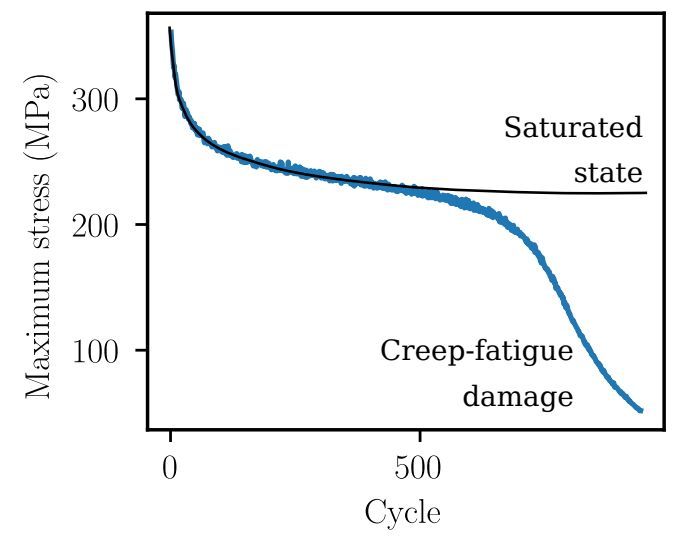

Figure 3.11: Experimental fatigue data (personal communication, M. Li, Argonne National Laboratory) for Grade 91 at $T=600^{\circ} \mathrm{C}$ overlaid with a hypothetical saturated state.

of cyclic flow stress. This extrapolation intentionally ignores the later, damaged induced softening.

We propose to use this hypothetical saturated state to determine saturated, "bounding" values of the cyclic softening coefficient. These values are not strictly bounding - after all the flow stress in actual experiments continues to decrease. However, we argue that the creep-fatigue design rules, discussed in the next Chapter, will guard against the material ever reaching the terminal, non-saturating, damage-induced portion of the cyclic softening curve. This approach, described also in a previous report, forms the basis of the recommended modifications to the existing EPP Code Cases, described in Chapter 5. 


\section{Creep-fatigue}

This chapter compares the EPP creep-fatigue Code Case N-862 [4] to the results of a consistent full inelastic simulation. Figure 4.1 shows the reference component used for this comparison. The component has the same geometry and similar loading conditions to the component used for the evaluation of the strain limits procedure in Chapter 3. However, in these simulations both the temperature and the pressure are cycled in phase. The consistent comparison process is essentially the same as for the evaluation of the ratcheting strain design provisions. The simulations first apply $n_{\text {pre }}$ cycles of prior, pure fatigue loading. In the inelastic simulations the full load cycle, including hold, is repeated until the damage model predicts the component fails.

The corresponding EPP calculations use a pseudoyield stress to bound creep damage in the structure. This pseudoyield stress is based on a trial design life and the design rupture lives, here established in Chapter 2. A strain range is extracted from the resulting EPP analysis, which can be used to evaluate fatigue damage using the design fatigue curves also described in Chapter 2. Finally, the values of creep and fatigue damage can be entered into the design D-diagram and the structure evaluated to see if it passes the creep-fatigue design evaluation.

All this design data was generated for the reference inelastic model in Chapter 2 for material in the as-received condition.

Figure 4.2 compares the creep-fatigue design life calculated with the reference inelastic model to the design life calculated from the unmodified EPP creep-fatigue Code Case. The figure shows that the creep-fatigue life calculated with the Code Case EPP procedure is conservative for any number of prior fatigue cycles. This indicates that that unmodified method, established by Code Case N-862, should be conservative without modification, even for a cyclic softening material like Grade 91 . This is in contrast to the strain limits procedure, which required a modification to account for prior cyclic softening.

Note the EPP design life becomes zero at around 250 cycles of pure fatigue loading. This is because the EPP method predicts failure even when only applying the fatigue pre-cycles.

The reason why the creep-fatigue EPP design procedure does not require a modification to account for cyclic softening is that the D-diagram already tacitly bounds the effects of cyclic softening on creep-fatigue life. Recall that the damage diagram is constructed by plotting the results of many creep-fatigue tests, in terms of their computed fatigue and creep damage ratios, and drawing a design envelope through the data.

By definition these creep-fatigue tests include the effect of cycling on creep life (and viceversa). If cycling softens the material and accelerates the creep rates, like Grade 91, the creep-fatigue experiments will capture this effect.

This explains why the creep-fatigue interaction for Grade 91 is so severe. The ASME Section III, Division 5 diagram for Grade 91 uses an intersection point of $(0.1,0.01)$ - by far the most severe creep-fatigue interaction of any of the Class A materials. We argue that this severe creep-fatigue interaction reflects the underlying material cyclic softening causing elevated creep rates for fatigue-dominated loading, severely limiting the allowable creep damage. 

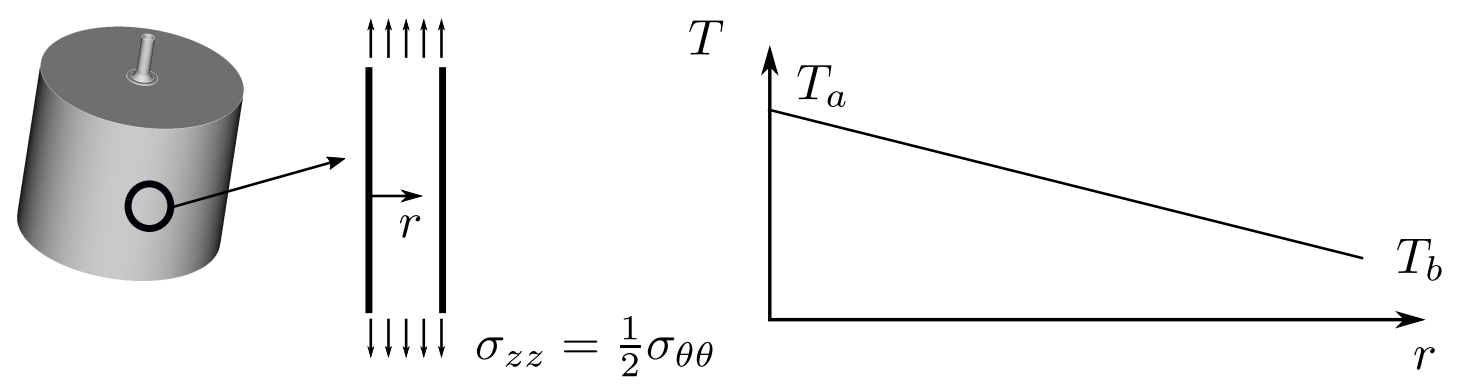

Linear through-wall thermal gradient

Full load cycle

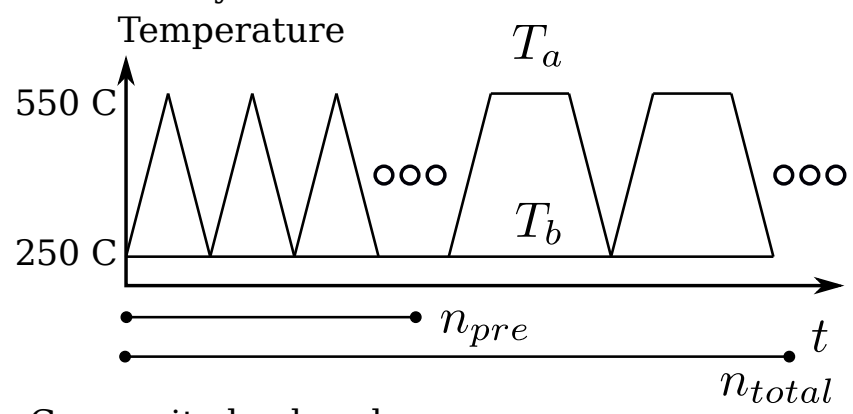

Hoop stress

Composite load cycle

Temperature
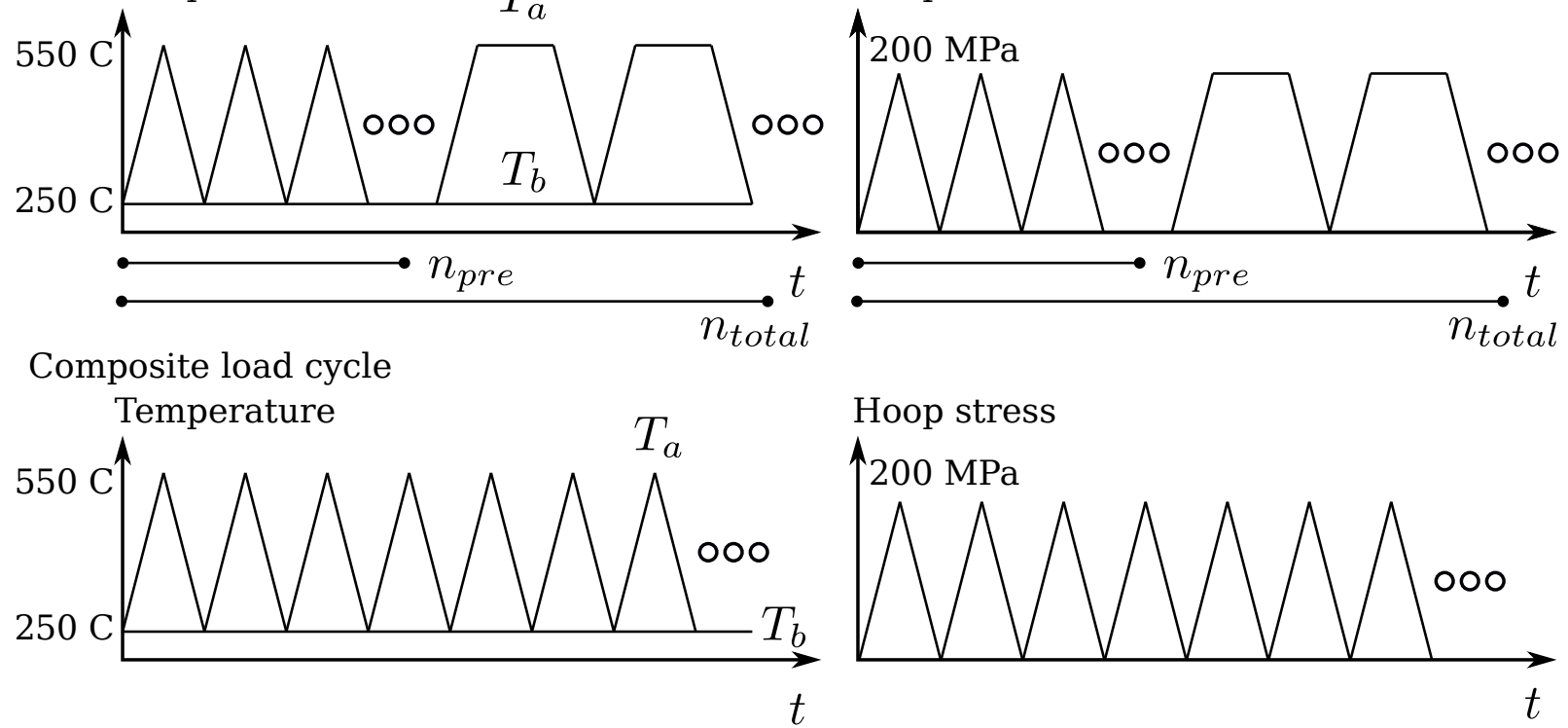

Hoop stress

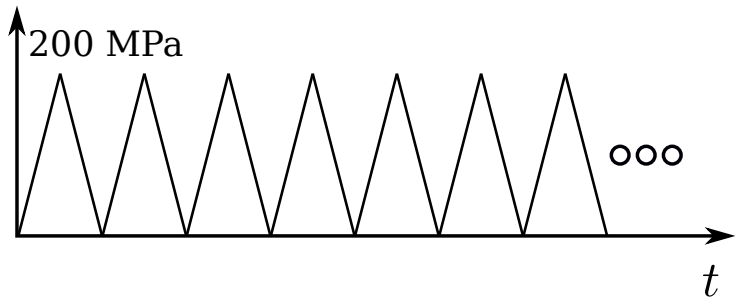

Figure 4.1: Sketch illustrating the reference component used to assess the EPP creep-fatigue design method. 
Initial development and extension of EPP methods to Grade 91

July 2018

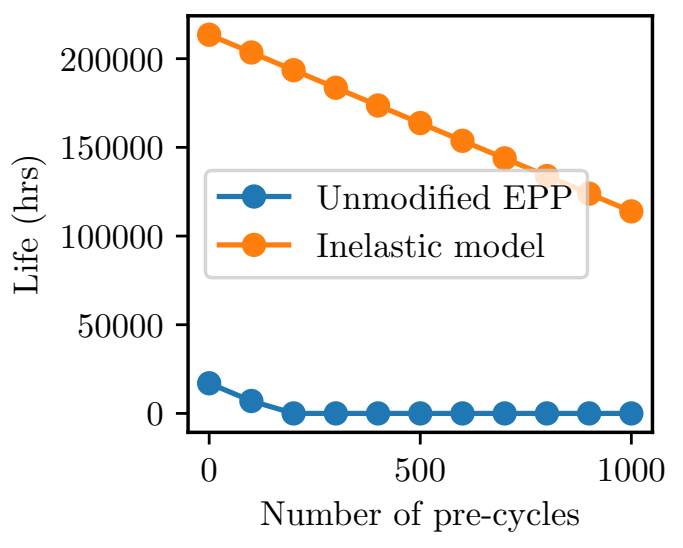

Figure 4.2: Plot comparing the design lives predicted by the unmodified EPP creep-fatigue design procedure to the reference inelastic model. 



\section{Proposed modifications to the EPP Code Cases}

This chapter proposes modifications to the EPP Code Cases for austenitic steel to make them suitable for use for Grade 91.

\subsection{Strain limits: Code Case N-861}

Chapter 3 proposes a technical change to the EPP method described in Code Case N-861 which accounts for cyclic softening by using reduction factors applied to the material yield stress $S_{y}$ and the values of the isochronous curve when these quantities are used to calculate the pseudoyield stress for the EPP bounding procedure.

A prior report [21] described the process of developing softening factors for the isochronous curves in greater detail. In addition to deriving softening factors based on a reference inelastic model (that did not include damage) that report also described how to experimentally measure softening factors using creep and creep-fatigue experiments. Table 5.1 reproduces the experimental and model-based softening factors from the prior report. The table includes bounding softening factors for both 300,000 and 500,000 hours maximum design lives. The table also lists a suggested softening factor as a function of temperature based on the available data. This table covers the creep range for Grade 91, as defined by Section III, Division 5. The model predicted very rapid softening for the material at $650^{\circ} \mathrm{C}$. A softening factor of zero effectively bounds those results. Softening factors might be reasonably interpolated in between table values.

Previous work has not considered corresponding softening factors for the material yield stress $S_{y}$. However, the required experimental data is available in the literature in the form of strain-controlled cyclic tests on the material. Table 5.2 suggests softening factors to be applied to $S_{y}$ based on the available experimental data extrapolated out to the saturated cyclic state. Figure 5.1 shows an example of how this extrapolation was performed for the available data at room temperature. The table cites the experimental data used to determine the softening factor at each temperature. As with the isochronous curve softening factors it would be reasonable to interpolate in between table values.

Several changes would be required to the Code Case N-861 language to include the softening factors for Grade 91 steel. Changes in the following quotes from the existing Code Case are emphasized with italics.

The inquiry and reply would need to be altered to allow Grade 91:

\begin{tabular}{lllll}
\hline Temperature $(\mathrm{C})$ & Recommended & $\begin{array}{l}\text { Model factor } \\
(300,000 \text { hours })\end{array}$ & $\begin{array}{l}\text { Model factor } \\
(500,000 \text { hours })\end{array}$ & \begin{tabular}{l} 
Experimental factor \\
\hline 375
\end{tabular} \\
\hline 0.85 & 0.87 & 0.87 & - \\
500 & 0.83 & 0.85 & 0.85 & - \\
600 & 0.77 & 0.79 & 0.82 & - \\
650 & 0.70 & 0.72 & 0.71 & 0.68 \\
\hline
\end{tabular}

Table 5.1: Table showing the recommended, model-based, and, where available experimental isochronous curve softening factors for Grade 91. 


\begin{tabular}{lll}
\hline Temperature $(\mathrm{C})$ & Factor & Sources \\
\hline 25 & 0.88 & {$[24]$} \\
500 & 0.84 & {$[15,18]$} \\
600 & 0.72 & {$[15,18]$} \\
650 & 0.62 & {$[15]$} \\
\hline
\end{tabular}

Table 5.2: Table showing the recommended softening factors for $S_{y}$ for Grade 91.

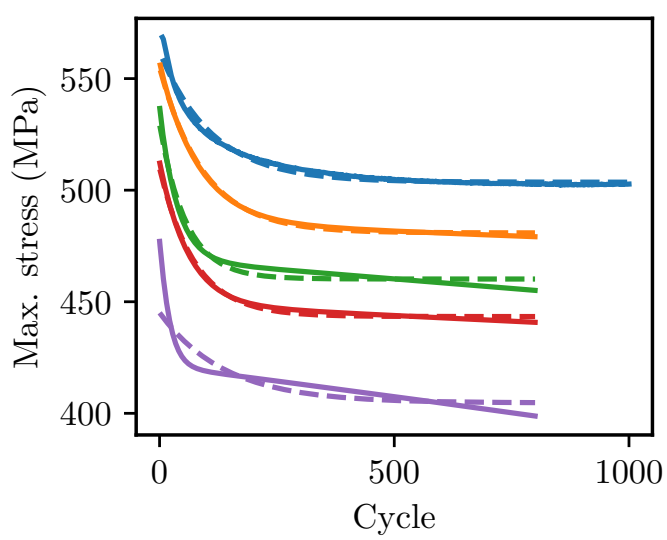

Figure 5.1: Figure showing how the experimental data (solid lines) was extrapolated out to a saturated state (dashed lines).

Inquiry: What alternative rules may be used for the evaluation of strain limits in compliance with Section III, Division 5, Subsection HB, Subpart B, HBB-3252 and Nonmandatory Appendix HBB-T?

Reply: Strain limits for $9 C r-1 M o-V$ per Section III, Division 5, Table HBB-I-14.1(a) may be evaluated using elastic-perfectly plastic material models instead of the procedures of Section III, Division 5, HBB-T-1320, HBB-T-1330, and HBB-T-1713 when performed in accordance with the requirements of this Code Case.

The analysis steps in Section 4 of the Code Case would need to be altered to implement the softening factors:

Step 2. Select a target inelastic strain, $x$, where $0<x<\varepsilon_{\text {avg }}$, and $\varepsilon_{\text {avg }}$ is equal to 0.01 for base metal or 0.005 for weldments. Define a pseudo-yield stress, $S_{x T}$, at each location, using the temperature determined from the transient thermal analysis. This pseudo-yield stress is equal to the lesser of the quantities defined in (a) and (b) below.

(a) The yield strength, $S_{y}$, given in Section III, Division 5, Table HBB-I-14.5 multiplied by the cyclic softening factor given in Table 1.

(b) The stress to cause, $x$, inelastic strain in time $t_{\text {design }}$, as determined from the isochronous stress-strain curves in Section III, Division 5, Figure HBB-T-1800, multiplied by the cyclic softening factor given in Table 2.

Table 1 of the new Code Case would be Table 5.2 in this report and Table 2 would the 
recommended values from 5.1.

\subsection{Creep-fatigue: Code Case N-862}

As described in Chapter 4 the current Code Case N-862 [4] does not require any fundamental modifications to make it suitable for use in designing components made of Grade 91 steel. The only required change to the current Code Case would be to alter the inquiry and reply to allow designers to use the Code Case rules for Grade 91:

Inquiry: What alternative rules may be used for the calculation of creep-fatigue damage in compliance with Section III, Division 5, Subsection HB, Subpart B, HBB-3252 and Nonmandatory Appendix HBB-T?

Reply: Fatigue and creep damage may be evaluated for $9 \mathrm{Cr}-1 \mathrm{Mo}-\mathrm{V}$ per Section III, Division 5, Table HBB-I-14.1(a) using elastic-perfectly plastic material models instead of the procedures of Section III, Division 5, HBB-T-1420, HBB-T-1430, and HBB-T1715 when performed in accordance with the requirements of this Code Case.

Changes to the existing Code Case language are highlighted with italics. 



\section{Conclusions}

This report describes modifications to the existing EPP procedures for evaluating the Section III, Division 5 deformation controlled quantity design limits to account for cyclic softening in Grade 91 steel. The modified EPP methods conservatively bound ratcheting strain accumulation and creep fatigue damage in Grade 91 steel and can be implemented as a new ASME Nuclear Code Case.

The modified EPP strain limits method uses temperature dependent softening factors to account for cyclic softening in Grade 91. These factors are based on an extrapolated saturated, softened material condition. The actual material rarely reaches this condition because creep-fatigue damage begins to accumulate first, causing a further reduction in the flow stress. The ASME creep-fatigue rules guard against the development of creep-fatigue damage and so the EPP rules, taken as a whole, will prevent damage-induced softening from negatively affecting the proposed EPP strain limits procedure.

The analysis described in this report shows that no modifications are required to the existing EPP creep-fatigue design provisions. The D-diagram used to represent creep-fatigue interaction in the current rules already accounts for cyclic softening. Chapter 5 summarizes potential Code Cases to implement the EPP rules for Grade 91 steel.

This report then completes work on modifying the EPP Code Cases to apply to Grade 91 steel. Further work may be required to provide additional supporting evidence to ASME Code committees, but no substantial modifications to the design procedures are expected. Additional creep-fatigue experiments of the type described in Chapter 3 may be needed to validate the proposed isochronous softening factors for Grade 91. However, existing experimental data agree well with the factors derived from model predictions and so an extensive experimental program should not be required. 

Initial development and extension of EPP methods to Grade 91

July 2018

\section{Acknowledgments}

The research was sponsored by the U.S. Department of Energy (DOE), under Contract No. DE-AC02-06CH11357 with Argonne National Laboratory, managed and operated by UChicago Argonne LLC. Programmatic direction was provided by the Office of Advanced Reactor Technologies (ART) of the Office of Nuclear Energy (NE). 



\section{Bibliography}

[1] R. A. Ainsworth. Bounding solutions for creeping structures subjected to load variations above the shakedown limit. International Journal of Solids and Structures, 15(12):981986, 1979.

[2] R. A. Ainsworth. Appplication of bounds for creeping structures subjected to load variations above the shakedown limit. International Journal of Solids and Structures, 13:981-993, 1979.

[3] American Society of Mechanical Engineers. Case N-861: Satisfaction of Strain Limits for Division 5 Class A Components at Elevated Temperature Service Using Elastic-Perfectly Plastic Analysis. In ASME Boiler and Pressure Vessel Code, Nuclear Component Code Cases. 2015.

[4] American Society of Mechanical Engineers. Case N-862: Calculation of Creep-Fatigue for Division 5 Class A Components at Elevated Temperature Service Using ElasticPerfectly Plastic Analysis. In ASME Boiler and Pressure Vessel Code, Nuclear Component Code Cases. 2015.

[5] American Society of Mechanical Engineers. Section III, Division 5. In ASME Boiler and Pressure Vessel Code. 2017.

[6] ASTM International. E 21-09: Standard Test Methods for Elevated Temperature Tension Tests of Metallic Materials, 2009.

[7] P. Carter. Bounding theorems for creep-plasticity. International Journal of Solids and Structures, 21(6):527-543, 1985.

[8] Peter Carter. Analysis of cyclic creep and rupture. Part 1: Bounding theorems and cyclic reference stresses. International Journal of Pressure Vessels and Piping, 82(1): $15-26,2005$.

[9] Peter Carter. Analysis of cyclic creep and rupture. Part 2: Calculation of cyclic reference stresses and ratcheting interaction diagrams. International Journal of Pressure Vessels and Piping, 82(1):27-33, 2005.

[10] J. L. Chaboche. On some modifications of kinematic hardening to improve the description of ratchetting effects. International Journal of Plasticity, 7(7):661-678, 1991.

[11] J. L. Chaboche and G. Cailletaud. On the calculation of structures in cyclic plasticity or viscoplasticity. Computers and Structures, 23(1):23-31, 1986.

[12] J. L. Chaboche and D. Nouailhas. A unified constitutive model for cyclic viscoplasticity and its applications to various stainless steels. Journal of Engineering Materials and Technology, 111:424-430, 1989.

[13] J.L. Chaboche. Constitutive equations for cyclic plasticty and cyclic viscoplasticity. International Journal of Plasticity, 5:247-302, 1989. 
[14] B. Fournier, M. Sauzay, and A. Pineau. Micromechanical model of the high temperature cyclic behavior of $9-12 \% \mathrm{Cr}$ martensitic steels. International Journal of Plasticity, 27 (11):1803-1816, 2011.

[15] S Kim and J R Weertman. Investigation of Microstructural Changes in a Ferritic Steel Caused by High Temperature Fatigue. Metallurgical Transactions, 19A(April):999-1007, 1988.

[16] U F Kocks. Realistic constitutive relation for metal plasticity. Materials Science and Engineering A, 317:181-187, 2001.

[17] U. F. Kocks, A. S. Argon, and M. F. Ashby. Thermodynamics and kinetics of slip. In B. Chalmers, J. W. Christian, and T. B. Massalski, editors, Progress in Materials Science, pages 64-291. 1975.

[18] Gyeong H. Koo and Ji Hyun Kwon. Identification of inelastic material parameters for modified 9Cr-1Mo steel applicable to the plastic and viscoplastic constitutive equations. International Journal of Pressure Vessels and Piping, 88(1):26-33, 2011.

[19] F. R. Larson and J. Miller. A time-temperature relationship for rupture and creep stresses. Transactions of the ASME, 74:765-771, 1952.

[20] H Mecking, B Nicklas, N. Zarubova, and U F Kocks. A "universal" temperature scale for plastic flow. Acta Metallurgica, 34(3):527-535, 1986.

[21] M. C. Messner and T.-L. Sham. FY17 Status Report on the Initial EPP Finite Element Analysis of Grade 91 Steel. Technical report, Argonne National Laboratory, ANL-ART$94136949,2017$.

[22] M. C. Messner, V.-T. Phan, and T.-L. Sham. FY17 Status Report on the Initial Development of a Constitutive Model for Grade 91 Steel. Technical report, Argonne National Laboratory, 2017.

[23] Vani Shankar, M. Valsan, K. Bhanu Sankara Rao, R. Kannan, S. L. Mannan, and S. D. Pathak. Low cycle fatigue behavior and microstructural evolution of modified 9Cr-1Mo ferritic steel. Materials Science and Engineering A, 437(2):413-422, 2006.

[24] Kuo Zhang and Jarir Aktaa. Characterization and modeling of the ratcheting behavior of the ferritic-martensitic steel P91. Journal of Nuclear Materials, 472:227-239, 2016. 
Initial development and extension of EPP methods to Grade 91

July 2018

\section{Distribution List}

$\begin{array}{lll}\text { Name } & \text { Affiliation } & \text { Email } \\ \text { Caponiti, A. } & \text { DOE } & \text { alice.caponiti@nuclear.energy.gov } \\ \text { Gouger, H.D. } & \text { INL } & \begin{array}{l}\text { hans.gougar@inl.gov } \\ \text { cgrandy@anl.gov }\end{array} \\ \text { Grandy, C. } & \text { ANL } & \text { bobhill@anl.gov } \\ \text { Hill, R.N. } & \text { ANL } & \text { gkrumdick@anl.gov } \\ \text { Krumdick, G.K. } & \text { ANL } & \text { sue.lesica@nuclear.energy.gov } \\ \text { Lesica, S. } & \text { DOE } & \text { mli@anl.gov } \\ \text { Li, M. } & \text { ANL } & \text { michael.mcmurtrey@inl.gov } \\ \text { McMurtrey, M. } & \text { INL } & \text { messner@anl.gov } \\ \text { Messner, M.C. } & \text { ANL } & \text { natesan@anl.gov } \\ \text { Natesan, K. } & \text { ANL } & \text { quallsal@ornl.gov } \\ \text { Qualls, A.L. } & \text { ORNL } & \text { ssham@anl.gov } \\ \text { Sham, T.-L. } & \text { ANL } & \text { dsingh@anl.gov } \\ \text { Singh, D. } & \text { ANL } & \text { thomas.sowinski@nuclear.energy.gov } \\ \text { Sowinski, T.E. } & \text { DOE } & \text { wangh@ornl.gov } \\ \text { Wang, H. } & \text { ORNL } & \text { wangy2@ornl.gov } \\ \text { Wang, Y. } & \text { ORNL } & \text { richard.wright@inl.gov } \\ \text { Wright, R. } & \text { INL } & \text { xuanzhang@anl.gov } \\ \text { Zhang, X. } & \text { ANL } & \end{array}$






\section{Argonne}

\section{Applied Materials Division}

Argonne National Laboratory

9700 South Cass Avenue, Bldg. 208

Argonne, IL 60439

www.anl.gov 